\title{
Data-driven inference of network connectivity for modeling the dynamics of neural codes in the insect antennal lobe
}

\author{
Eli Shlizerman $^{1 *}$, Jeffrey A. Riffell ${ }^{2}$ and J. Nathan Kutz ${ }^{1}$ \\ ' Department of Applied Mathematics, University of Washington, Seattle, WA, USA \\ ${ }^{2}$ Department of Biology, University of Washington, Seattle, WA, USA
}

\author{
Edited by: \\ Florentin Wörgötter, University \\ Goettingen, Germany \\ Reviewed by: \\ Ramon Huerta, University of \\ California San Diego, USA \\ Shannon Bryn Olsson, National \\ Center for Biological Sciences, India \\ *Correspondence: \\ Eli Shlizerman, Department of \\ Applied Mathematics, University of \\ Washington, Box 353925, Seattle, \\ WA 98195 USA \\ e-mail: shlizee@uw.edu
}

The antennal lobe (AL), olfactory processing center in insects, is able to process stimuli into distinct neural activity patterns, called olfactory neural codes. To model their dynamics we perform multichannel recordings from the projection neurons in the AL driven by different odorants. We then derive a dynamic neuronal network from the electrophysiological data. The network consists of lateral-inhibitory neurons and excitatory neurons (modeled as firing-rate units), and is capable of producing unique olfactory neural codes for the tested odorants. To construct the network, we (1) design a projection, an odor space, for the neural recording from the $A L$, which discriminates between distinct odorants trajectories (2) characterize scent recognition, i.e., decision-making based on olfactory signals and (3) infer the wiring of the neural circuit, the connectome of the AL. We show that the constructed model is consistent with biological observations, such as contrast enhancement and robustness to noise. The study suggests a data-driven approach to answer a key biological question in identifying how lateral inhibitory neurons can be wired to excitatory neurons to permit robust activity patterns.

Keywords: data-driven modeling, reduced dynamics, olfactory neural coding, neuronal networks, odor discrimination, contrast enhancement, insect olfaction

\section{INTRODUCTION}

In the olfactory system, neural codes take the form of spatial firing-rate (FR) patterns exhibited by the output neurons of the neural processing unit, the antennal lobe $(\mathrm{AL})$ in insects and olfactory bulb (OB) in mammals (Laurent, 1999, 2002; Stopfer and Laurent, 1999; Galizia and Menzel, 2000; Riffell et al., 2009a). They were established by the application of standard data analysis techniques, e.g., Principal Components Analysis (PCA), to the time series of FR responses recorded from the output neurons (Broome et al., 2006; Harris et al., 2011). The success of these methods indicates that the response of cell assemblies is indeed low-dimensional so that for each individual stimulus a unique trajectory in a low-dimensional subspace is identified.

With these discoveries, it is intriguing to understand how sensory neural networks are designed to produce such behavior. Specifically, why do the encoding dynamics appear to be robust even for noisy stimuli? and what is the network architecture capable to produce these patterns (Wilson, 2008; Nagel and Wilson, 2011)? For the first question, investigations suggest that cell assemblies maintain several mechanisms for shaping the correct output response. One such mechanism is known to be lateral inhibition (Laurent, 1999; Egger et al., 2003), where both inhibitory and excitatory neurons receive common input and interact to mediate the response of excitatory neurons. A hallmark of lateral inhibition is contrast enhancement, which signature is an increase in signal to noise ratio, such that the amplitude or frequency of the response is easily distinguished from the response to random stimuli (Laughlin and Osorio, 1989; Yokoi et al., 1995; Cook and McReynolds, 1998; Olsen and Wilson, 2008; Wilson, 2008).

For the question of determining the network architecture that produces the neural codes it is required to model the actual network of neurons responsible for the encoding. The modeling procedure involves reconstruction of the network wiring, i.e., modeling individual neuron dynamics and their network interactions (connectome) (Seung, 2011; Jbabdi and Behrens, 2012). However, the connectome of different sensory neuronal networks may vary. For example, in vision, the retinal ganglion cells are ordered such that locally neighboring cells are responsive to neighboring parts of the visual stimulus, termed a retinotopic map (Bock et al., 2011). In olfaction, output neurons are also selective for certain odorant stimuli, providing a chemotopic map. However, the neighboring output neurons are not necessarily similar in their tuning to specific chemicals. Instead lateral inhibition mediates and shapes the responses of the output neurons, resulting in an effective chemotopic map between the input and the output cells (Cleland and Linster, 2005; Linster et al., 2005; Silbering and Galizia, 2007; Reisenman et al., 2008). Resolving this mapping is critical for determining how neurons process chemical information.

Inspired by the dimension reduction results and in order to find the mechanism responsible for the low dimensional dynamics several approaches were proposed to model the underlying neuronal network (reviewed in detail in Buckley and Nowotny, 2012). Afraimovich et al. (2004) used the top-down approach to construct the connectivity by restricting the network to 
have particular dynamics governed by low-dimensional homoclinic orbits deriving a 'winnerless' network able to produce robust transient dynamics in low-dimensional space (see also Rabinovich et al., 2001, 2006). Alternatively, Linster et al. (2005) used a bottom-up approach to derive a qualitative model for the honeybees AL where each neuron was modeled as a FR unit and the connectivity chosen to be inhibitory and drawn from a distribution that matches experimental knowledge (e.g., connecting pairs proportional to the similarity of their odor-response profiles). The model showed correspondence with experimental data when compared with calcium imaging dynamics suggesting that functionally organized inhibitory network, as opposed to anatomically structured network (local), all-to-all or random inhibitory network, best reproduces the input-output function of the AL. The use of firing-rate models was later justified by Buckley and Nowotny (2011) where it was shown that a network of Hodgkin-Huxley neurons can be reduced to firing-rate models and that stable fixed point dynamics are the most consistent with the FR time series data. While these works show that the AL is capable of extracting low dimensional features and can be modeled using firing rate units, the wiring of lateral inhibition within the models is set randomly. Therefore, the structural properties of the network that permit neural codes still remain unresolved (Rabinovich et al., 2008). Without resolving the network connectivity one cannot understand the observed features of odor processing such as contrast enhancement.

Electrophysiological recordings that sample the output from the AL could be potentially useful to infer candidates for such wirings. The most suitable approach for solving this inverse problem' would be the top-down approach, since it attempts to construct a low-dimensional model and establish the underlying mechanisms that determine network units and connectivity. However, it is currently unknown how to calibrate the low dimensional model using multi-neuron recordings (Mazor and Laurent, 2005; Rabinovich et al., 2008; Buckley and Nowotny, 2012). To overcome this difficulty, we propose the dynamical dimension reduction method that takes the top-down approach in conjunction with multineuron recordings. With this methodology we model the AL in the Manduca sexta moth a well-characterized physiological and behavioral experimental neural system in olfaction (Reisenman et al., 2008; Riffell et al., 2009a). The outcome of the approach is a high-dimensional system that exhibits lowdimensional dynamics. The method is fundamentally different than standard top-down approaches, as it does not determine parameters by simulation and fitting, which are biased by the simulations performed (e.g., choice of initial conditions), fitting of time-dependant signals and comparison metric. Instead, it projects a high-dimensional dynamical system onto orthogonal modes to be derived from data. This step is implicit and achieves a projected low dimensional system that is generic. As such it could be applicable for modeling the AL across different species and other neuronal networks. In the following step the projected dynamical system is matched with the conjectured lowdimensional dynamical system. We show that in the case of fixed points the matching can be formulated as a convex optimization problem. The system becomes explicit when the neural codes obtained from recordings are plugged-in and the optimization problem is solved to infer the wiring. For the AL, the matching is based on the characteristic that the projected dynamics onto the neural codes exhibit a trajectory toward a well separated fixed point for each stimulus, a consistent feature in experimental observations and used for modeling in Buckley and Nowotny (2012). Inputting the experimental neural codes as orthogonal modes and solving a minimization problem infers a suggested network wiring of the AL capable to encode given stimuli. The model is then compared with the experimental dynamics for consistency. Our results show that the wirings obtained using such an approach produce neural codes that are unsusceptible to noise and thus suggest that the introduced methodology can assist in resolving the architecture of the AL and circuit-level properties.

\section{MATERIALS AND METHODS}

\subsection{DATA DRIVEN TOP-DOWN MODELING APPROACH}

The neural cell assemblies participating in the processing of olfactory information in the AL are the receptor cells (RNs) that carry the input from the environment, the projection (output) neurons (PNs), and local interneurons (LNs) (reviewed by Hildebrand and Shepherd, 1997; Hansson and Anton, 2000; Martin et al., 2011). We model the network by three vectors $\vec{x}, \vec{y}$, and $\vec{z}$, where each element in the vector represents a neuron and modeled by a firing-rate unit (Linster et al., 2005; Capurro et al., 2012; Chong et al., 2012). The three vectors correspond to the three anatomical groups RNs, PNs, and LNs, respectively:

$$
\begin{aligned}
& \dot{\vec{x}}=-\vec{x}+\vec{J}, \\
& \dot{\vec{y}}=-\beta \vec{y}+[A \vec{x}-B \vec{z}]^{+}, \\
& \dot{\vec{z}}=-\gamma \vec{z}+[C \vec{x}-E \vec{z}]^{+}
\end{aligned}
$$

The input into the PNs and LNs is modulated by a standard linear threshold function denoted by [.] ${ }^{+}$, as in Linster et al. (2005) and Buckley and Nowotny (2012). Figure 2A illustrates the threshold function used here. The vector $\vec{J}$ is the external input into the RNs which is driven by the chemosensory processes in the antenna.

In the deterministic version of this model, where the input is either constant or time dependant, the dynamics can be intuitively described. Specifically, when there is significant input into the population of receptor neurons $(\vec{x})$, these neurons lock onto the driving input $\vec{J}$ (Buckley and Nowotny, 2011, 2012). In the case of constant input, the receptor population will converge to a fixed point $\vec{x}_{0}=\vec{J}$. This in turn excites both the projection neurons $(\vec{y})$ and the interneuron populations $(\vec{z})$. A meaningful input should excite a spatial stable pattern $\vec{y}^{P}$ in the projection neurons. The stable spatial patterns $\vec{y}^{P}$ are thought of as library elements which encode various recognized odorants. Note that the pattern is not necessarily equal to the input, i.e., $\vec{y}^{P} \neq \vec{J}$.

Our goal is to understand how the network in Equations (1-3) can be made capable to produce stable patterns and discriminate between them. Particularly, we would like to find a network connectome, consisting of the connectivity matrices $A, B, C$, and $E$, that enhances the components in the input that correspond to recognized patterns $\left(\vec{y}^{P}\right)$ and inhibits other remaining components. In practice, the structure of the connectivity matrices $A$ and $C$ is local and can be obtained from anatomical experimental 
knowledge, while the structure of the matrices $B$ and $E$ is mostly unknown.

The method dynamical dimension reduction that we introduce in this work provides a procedure to construct the unknown matrices $B$ and $E$. The first step in the method is to obtain population encoding vectors (orthogonal patterns $\vec{y}^{P}$ ) from the electrophysiological recordings of $\mathrm{PN}$ neurons. We then show that a projection of the PN dynamical equations, Equation (2), onto the population encoding vectors provides a division of these equations into two models: a reduced model for the dynamics of population vectors and a model for the dynamics of remaining patterns. Separating the system into two models allows us to impose constraints on the dynamics of each model. Particularly, we require stable patterns in the reduced model and rapid decay of the remainder. We show that these requirements form a convex minimization problem which solution is the unknown connectome.

The projection is done as follows. If the system does not saturate, then the excitable regime can be modeled by a linear version of Equations (2-3) in which the brackets from the saturation terms are removed. Additionally, if the $\vec{x}$ dynamics are fast in comparison to those of $\vec{y}$ and $\vec{z}$ (RNs drive the response in LNs and PNs) (Geiger et al., 1997; Meyer et al., 2013), then $\vec{x}$ can be replaced by the input $\vec{J}$, i.e., its fixed point, and we derive the following system

$$
\begin{aligned}
& \frac{d \vec{y}}{d t}=-\beta \vec{y}+A \vec{J}-B \vec{z}, \\
& \frac{d \vec{z}}{d t}=-\gamma \vec{z}+C \vec{J}-E \vec{z} .
\end{aligned}
$$

In this system, the vector $\vec{y}(t)$ describes the dynamics of the coefficients of a standard basis $\left[y_{i}(t)\right.$ is the dynamics of $i$-th PN neuron]. However, we are interested in determining the dynamics of the observed patterns. From this representation, it is not immediately clear how to conclude which coding patterns in $\vec{y}$ appear while others do not, and what kind of connectivity matrices support such formations. Thus, the next step in our analysis is to decompose the system into encoding patterns and the remainder. For such a decomposition, we assume that there is a library matrix $L$ of observed patterns $L=\left\{\vec{y}_{1}^{P}, \ldots, \vec{y}_{l}^{P}\right\}$. We take into account that the library is a semi-positive matrix and we normalize each column vector (pattern) in the matrix. We then transform the matrix to an orthonormal matrix $O^{P}$. In this matrix, each column vector is called a population encoding vector and represents neurons and their expected firing-rates evoked by a particular input-key. The transformation to the orthonormal matrix is achieved by applying a threshold and a maximum rule on each element $l_{i j}$ of the matrix $L$. Thereby each element $o_{i j}^{P}$ in the matrix $O^{P}$ is defined as follows

$$
o_{i j}^{P}=U_{1}\left(l_{i j}\right)= \begin{cases}l_{i j} & \text { if } l_{i j}=\max \left(\vec{l}_{i}\right) \geq \tau . \\ 0 & \text { otherwise }\end{cases}
$$

where $\tau$ is the threshold value (chosen as $\tau=0.07$ in Figure 5). This construction results in a matrix with a single positive element in each row vector or a zero row vector, such that the system is effectively made orthogonal. The zero row vectors indicate PN neurons that do not substantially contribute to any of the patterns and thus these neurons will be considered to belong to the remainder vector. To construct the remainder vector, $\vec{o}^{R}$, we define the transformation $U_{2}$

$$
\vec{o}^{R}=U_{2}\left(U_{1}\left(l_{i j}\right)\right)=\left\{\begin{array}{l}
1 \text { if } \max \left(\vec{l}_{i}\right)=0 \\
0 \text { otherwise }
\end{array}\right.
$$

that assigns the value of unity if the corresponding row in $O^{P}$ that is a zero vector. As a final step we normalize $\vec{o}^{R}$ and augment the matrix $O^{P}$ with the vector $\vec{o}^{R}$ to create the matrix $O$ :

$$
L=\left[\begin{array}{ccc}
\vdots & & \vdots \\
\vec{y}_{1}^{P} & \ldots & \vec{y}_{l}^{P} \\
\vdots & & \vdots
\end{array}\right]_{N \times l} \rightarrow^{U} O=\left[\begin{array}{cccc}
\vdots & & \vdots & \vdots \\
\vec{o}_{1}^{P} & \ldots & \vec{o}_{l}^{P} & \vec{o}^{R} \\
\vdots & & \vdots & \vdots
\end{array}\right]_{N \times l+1}
$$

This allows us to describe the dynamics of PNs with the following low rank decomposition

$$
\begin{array}{r}
\vec{y}(t)=p_{1}(t) \vec{o}_{1}^{P}+\ldots+p_{l}(t) \vec{o}_{l}^{P}+r(t) \vec{o}^{R} \\
=O\left(\begin{array}{c}
p_{1}(t) \\
\vdots \\
p_{l}(t) \\
r(t)
\end{array}\right)=O \vec{p}
\end{array}
$$

Here we multiply each of the population vectors (stationary) by a dynamical coefficient $p_{j}(t)$ and the remainder population vector by $r(t)$. To derive the equations for the dynamics of the coefficients $\vec{p}(t)$, we substitute the decomposition of Equation (6) into Equation (4) and multiply the equations for $\vec{y}$ by the transpose matrix $O^{T}$ and use the fact that for semi-orthogonal matrices $O^{T} O=I$. Thus,

$$
\begin{aligned}
& \frac{d \vec{p}}{d t}=-\beta \vec{p}+O^{T}(A \vec{J}-B \vec{z}), \\
& \frac{d \vec{z}}{d t}=-\gamma \vec{z}+C \vec{J}-E \vec{z} .
\end{aligned}
$$

This projection technique is based on the Proper Orthogonal Decomposition method introduced in Sirovich $(1987,1996)$ and applied to reduction of neuronal networks in Shlizerman et al. (2012).

In this section we consider the case where the input is timeindependent and in the Results section explore the system dynamics with time dependent and noisy inputs. Since the dynamics in $\vec{z}$ are independent of the dynamics in $\vec{p}$, we can solve the second equation in Equation (7) for a fixed point $(d \vec{z} / d t=0)$

$$
\vec{z}_{0}=\tilde{E}^{-1} C \vec{J}, \quad \tilde{E}=E+\gamma I
$$

Then plugging-in into the first equation the expression of the fixed point we receive 


$$
\begin{aligned}
& \frac{d \vec{p}}{d t}=\tilde{M} \vec{p}+\vec{J}^{e f f} \\
& \tilde{M}=-\beta I, \vec{J}^{e f f}=O^{T}\left(A-B \tilde{E}^{-1} C\right) \vec{J} .
\end{aligned}
$$

The resulting reduced system is a linear inhomogeneous system of ODEs. For constant inputs, LNs will eventually equilibrate to the fixed point $\vec{z}_{0}$, determined by the values of $\gamma$ and $E$. For pulsed stimuli, which are often considered in experiments (e.g., the duration of stimulation used here is $200 \mathrm{~ms}$ ), LNs typically respond to the onset of stimulus with bursting and release fast GABA-A transmitters (within 1-2 ms) (Christensen et al., 1998) and later with slower GABA-B transmitters $(100 \mathrm{~ms})$ and are represented by the matrix $E$. Since there is a separation of time-scales between these types of transmitters, as a first order approximation the matrix $E$ represents fast connections, although both timescales can be incorporated.

The system in Equation (8) has terms that include the parameter $\vec{p}$ (multiplied by $\tilde{M}$ ) and non-homogeneous terms that are the effective input. Note that since there is no input from $\vec{y}$ into $\vec{z}$ (PN to LN) in Equations (4-5), the homogeneous term is multiplied by a diagonal matrix $\tilde{M}$. The matrix $\tilde{M}$ has only negative eigenvalues $\left(\lambda_{i}=-\beta\right)$ and thus by Lyapunov's stability theorem the model in Equation (8) is globally asymptotically stable, i.e, the system will always converge to a stable equilibrium $\vec{p}_{0}=(1 / \beta) \vec{J}^{\text {eff }}$ (Gajic and Lelic, 1996), see Figure 2C. In systems which have additional input from the $\vec{y}$ population into the $\vec{z}$ population, the matrix $\tilde{M}$ will involve non-diagonal terms that express interactions of the patterns. For such wirings it should be verified that the system is stable, i.e., the dynamics are as in Figure 2C, by solving the Lyapunov equation that will involve the connectivity matrices (Gajic and Lelic, 1996). The solution of the equation, if exists, will impose constraints on the configuration of the connectivity matrices such that the fixed point is stable. These constraints will be added to the optimization problem (11).

While the stability theorem assures that the dynamics of the patterns converge to an equilibrium, it does not guarantee separation of equilibria, which is required for a robust encodingdecoding system. Moreover, the matrices $B$ and $\tilde{E}$ are unknown, both in theory and in practice. For that purpose we need to calibrate the effective input into the population encoding vectors. Following the same procedure as for the output patterns we construct an orthogonal library matrix, $J_{0}$, for the input keys. Then the calibration is reduced to solving the following system of underdetermined equations

$$
O^{T}\left(A-B \tilde{E}^{-1} C\right) J_{0}=W
$$

with the prescribed matrix $W$ of dimensions $(l+1) \times(l+1)$ representing the calibration, and $B$ and $\tilde{E}$ are the unknown matrices. Essentially, this is a linear system of equations with a specified right hand side matrix $W$ where the matrix elements of $W$ determine physiologically relevant characterization of the importance of various odors. This is a highly undetermined set of equations that allows for an infinite number of solutions, i.e., there are an infinite number of ways to specify $B$ and $\tilde{E}$. Imposing further biophysical constraints could allow to obtain a unique biophysical solution.
Each row in $W$ encodes the effect of the different input keys, including the remainder, on a particular population encoding vector. For example, the element on the $i$-th row and $k$-th column, $w_{i, k}$, defines how $\vec{J}_{k}$ excites or inhibits $p_{i}(t)$. The elements of $W$ are set as follows

$$
W=\left[\begin{array}{cccc}
\ddots & & & \\
\hline & w_{i, i} & w_{i, k} & w_{i, l+1} \\
\hline & \ddots & \\
\hline w_{l+1,1} & & w_{l+1, k} & w_{l+1, l+1}
\end{array}\right] .
$$

The diagonal element on the $i$-th row, $w_{i, i}$, defines how $\vec{J}_{i}$ affects $p_{i}(t)$, its corresponding population encoding vector, and has to be set as positive (excitatory). The input from the other keys, $\vec{J}_{k}$, $k \neq i$, is encoded by $w_{i, k}$ and can be set 0 or negative. The input from the last key is the input from the remainder and is encoded by $w_{i, l+1}$. The value of this element should be strictly set to 0 , such that the remainder does not have excitatory or inhibitory effect on the population encoding vector. The last row in $W$ denotes the input into the remainder and thereby the elements, except the diagonal element on that row should be always negative. See the caption of Figure 2 for a possible configuration of the matrix $W$.

When $A$ and $C$ are known matrices, then the calibration is accomplished by solving an inverse problem to find the matrices $\tilde{E}$ and $B$ that satisfy these equations. Notice that the equations are underdetermined, i.e., the dimensions of $W$ are much lower than of $B \tilde{E}^{-1}$, indicating that the matrices $B$ and $\tilde{E}$ that satisfy Equation (9) are non-unique. To find the appropriate candidates for the matrices, we reformulate the inverse problem as a minimization problem

$$
\begin{gathered}
\operatorname{minimize}\left\|O^{T}\left(A-B \tilde{E}^{-1} C\right) J_{0}-W\right\|_{F r} \\
\text { subject to } \quad B, E \geq 0,
\end{gathered}
$$

where $\|\cdot\|$ is the Frobenius matrix norm. When the lateral connections between PNs and LNs are exclusively inhibitory the matrices $B, \tilde{E}$ are non-negative. When one of the matrices is set to particular wiring (e.g., $\tilde{E}$ is random) we need to determine only one matrix and the minimization problem is a semi-definite convex minimization. When there are excitatory lateral connections or the zero minimum cannot be attained, the semi-definite constraint is relaxed. Another possibility for negative terms in $B$ and $\tilde{E}$ is when the input keys and the output codes differ from each other in dimensions. Indeed, the matrices $B$ and $\tilde{E}$ permute the lateral effect of the interneurons to support such a coding scheme. Due to many degrees of freedom in the problem, additional constraints can be added. For example we can restrict the magnitudes of the elements in $B$ and $\tilde{E}$ not to exceed a particular value. Moreover, the calibration is particular to the choice of the matrices $A$ and $C$ (see an example in Figure 2B). To solve the minimization problem (11) or its variants, we employ the disciplined convex optimization package CVX implemented in MATLAB Grant and Boyd (2011).

For input keys being identical to the output population vectors, i.e., $J_{0} \equiv O$, the calibration creates a system that for a 
significant magnitude of one of the input keys, noise, and other population encoding vectors will be suppressed to allow for a decoding of the input-key (see Figure 4). Effectively this is a mechanism that produces contrast enhancement as we discuss in the Results section.

\subsection{ELECTROPHYSIOLOGICAL PREPARATION AND STIMULATION}

Manduca sexta L. (Lepidoptera: Sphingidae) male larvae were obtained from the Manduca-rearing facility of the Department of Biology of the University of Washington. Larvae were reared on artificial diet (Bell and Joachim, 1976) under long-day light:dark (LD) regimen $(\mathrm{LD} 17: 7)$ at $25-26^{\circ} \mathrm{C}$ and $40-50 \%$ relative humidity $(\mathrm{RH})$, and prepared for experiments $2-3 \mathrm{~d}$ after emergence. In preparation for electrophysiological recording, the moth was secured in a plastic tube with dental wax, leaving the head, and antennae exposed. The preparation was oriented so that both ALs faced upward, and the tracheae and sheath overlying one AL were carefully removed with a pair of fine forceps. The brain was superfused slowly with physiological saline solution throughout the experiment.

Electrophysiological recordings were made with 16-channel silicon multielectrode recording arrays (a4 × 4-3 mm 50-177; NeuroNexus Technologies, Ann Arbor, MI, USA). This microprobe allows the recording of neurons throughout the AL because of the probes dimensions, with four shanks spaced $125 \mu \mathrm{m}$ apart, each with four recording sites $50 \mu \mathrm{m}$ apart (Christensen et al., 2000; Riffell et al., 2009b). The probe was positioned under visual control using a stereo microscope. We use routine histological methods (e.g., Riffell et al., 2009a) to visualize the tracks left by the probes and identify the recording sites. Neural ensemble activity was recorded simultaneously from the 16 channels of the recording array using a RZ2 base station (Tucker-Davis Technologies, Alachua, FL, USA) and a PZ2 peamplifier. Spiking data from 16 channels (recorded at four sites on each of the 4 probes) were extracted from the recorded signals and digitized at $25 \mathrm{kHz}$ using the Tucker-Davis Technologies data-acquisition software. Spike data were extracted from the recorded signals in the tetrode configuration and digitized at $25 \mathrm{kHz}$ per channel. Filter settings (typically $0.6-3 \mathrm{kHz}$ ) and system gains (typically 5000-20,000) were software adjustable on each channel. Spikes were sorted using a clustering algorithm based on the method of principal components (PCs). Only those clusters that were separated in three dimensional space (PC1-PC3) after statistical verification (multivariate ANOVA; $P<0.05$ ) were used for further analysis (6-15 units were isolated per ensemble; $n=11$ ensembles in as many animals). Each spike in each cluster was time-stamped, and these data were used to create raster plots and to calculate the instantaneous firing-rates (iFRs). Based on the spiking activity, recorded spike trains were identified as an LN or PN (as in Brown et al., 2004; Riffell et al., 2009a, 2013; Lei et al., 2011). All analyses were performed with Neuroexplorer (Nex Technologies, Winston-Salem, NC, USA), or MATLAB (The Mathworks, Natick, MA, USA), using a bin width of $5 \mathrm{~ms}$, unless noted otherwise.

Olfactory stimuli were delivered to the preparation by pulses of air from a constant air stream were diverted through a glass syringe containing a piece of filter paper bearing floral odors. The stimulus was pulsed by means of a solenoidactivated valve controlled by the acquisition software (TuckerDavis Technologies, Alachua, FL, USA). AL neurons were stimulated with two pairs of odorants: (1) pair: "A": $\beta$-myrcene, a plant-derived odorant used to attract moths (Riffell et al., 2009a), "B": E10,Z12-hexadecadiennal (bombykal $\{\mathrm{Bal}\}$ ), the primary component of the conspecific females sex pheromone (Tumlinson et al., 1989, 1994). (2) pair: “C” BEA-benzaldehyde, and "D": BOL-benzyl alcohol. Stimulus duration was $200 \mathrm{~ms}$, and five pulses were separated by a $10 \mathrm{~s}$ interval. The stimulus durations reflect the time periods in which moths encounter odors when flying in their natural environment (Murils and Jones, 1981; Riffell et al., 2008), and the odorants used to stimulate the preparation are behaviorally effective stimuli, thus allowing neurobiological experimentation in a naturalistic context for discovering how neural circuits process odor information.

\section{RESULTS}

To study the AL's neural encoding dynamics we computationally model the AL as a network with each neuron modeled as a FR unit. In keeping with the populations of AL cells, three populations of FR units are considered: RNs that carry the input from the periphery (RNs), projection (output) neurons (PNs), and local inhibitory interneurons (LNs) (Figure 1). The dynamics of the populations are represented by the state vectors, $\vec{x}, \vec{y}$, and $\vec{z}$ corresponding to dynamics of RNs, PNs, and LNs, respectively. Each FR unit in each population is modeled by a differential equation that describes unit's self-dynamics (decay in the absence of input), interaction with other units and response to odorant stimulus (for a detailed description of the construction see the Materials and Methods section). The network can be calibrated to perform encoding functions, i.e., produce neural codes. Specifically, for each FR pattern that the PNs population exhibits (called population encoding vector), there is a FR pattern of the RNs population that evokes it (called input key) (depicted in Figures 1, 2). The results that we obtain from constructing the network establish how neurons' connectivity and network dynamics are linked together to produce these encoding functions. Analyzing computational dynamics and comparing them with experimental dynamics elucidates what are the typical dynamics of neural codes and how they can be perceived. We describe our results in detail below.

\subsection{RECOVERING THE CONNECTOME OF AN EXAMPLE NETWORK}

As an illustrative example of the theoretical construct proposed here, we demonstrate how we establish the neuronal wiring on a network of 10 neurons of each type: 10 RNs, 10 PNs, and 10 LNs for a total of 30 neurons. The network is designed to encode two input keys into two output population encoding vectors (codes) identical to the input keys. The goal of the calibration is to determine the connectivity matrix $B$ given the matrices $A, C$, and $E$ (Figure 1C). Specifically, we choose the matrices $A$ and $C$ to be identity matrices, i.e., each receptor is connected to its corresponding $\mathrm{PN}$ and $\mathrm{LN}$. The matrix $E$ is set as a random matrix whose elements are drawn from a uniform distribution with mean 0.25 , i.e., the LNs are randomly connected between themselves. We then solve an optimization problem, Equation (11), 


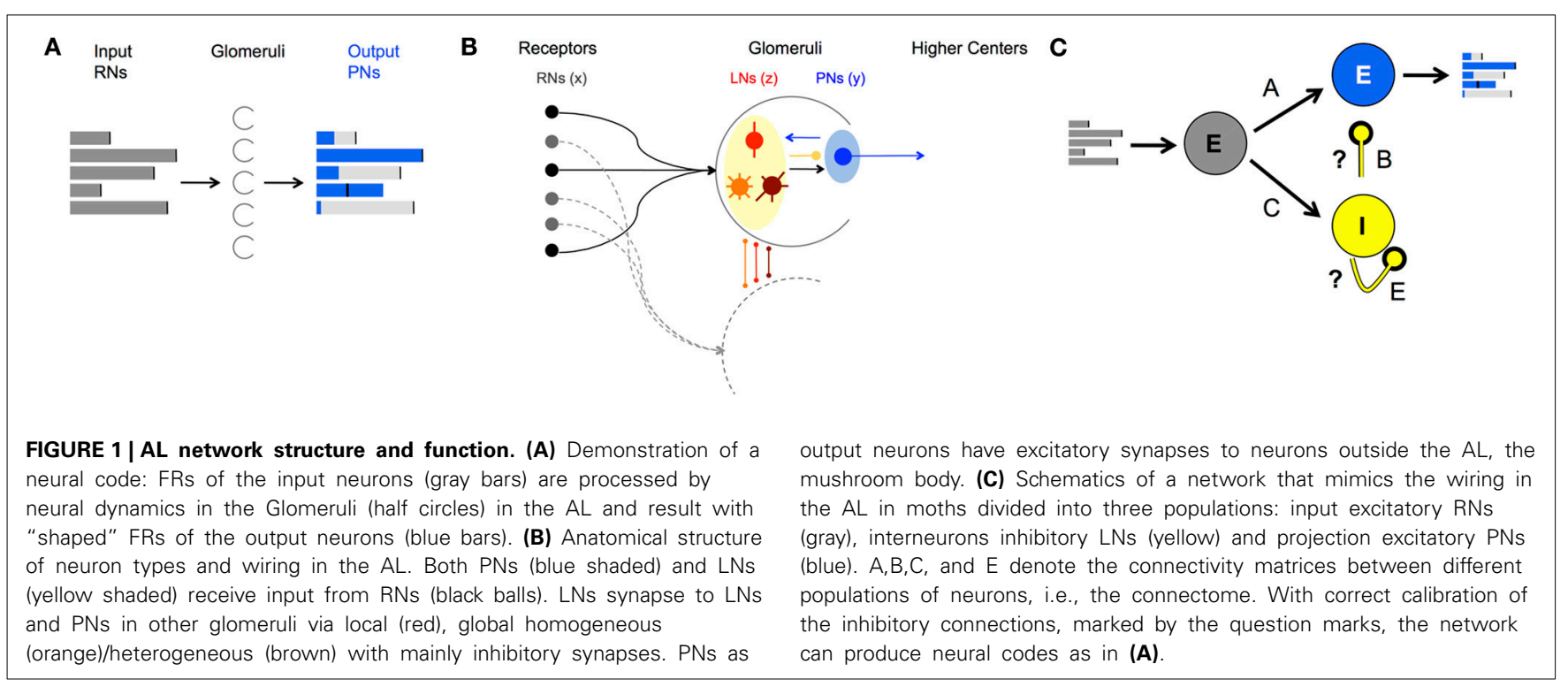

derived in the Materials and Methods section, to determine the elements of the matrix $B$. This is the optimal matrix that supports such an input-output relation (Figure 2B). The matrices are asymmetric, showing that our approach is consistent with experimental anatomical data. Moreover, it is fundamentally different than the Hopfield-type approach that uses symmetry constraint for optimization (Hopfield and Tank, 1986; Reisenman et al., 2008).

The calibration process produces connectivity matrices from which the connectome of the full network is recovered. To visualize the connectome we use the CIRCOS package (Krzywinski et al., 2009) where the network is depicted in a ring shape: FR units are drawn as arcs on the ring's perimeter and the connections are the links between the arcs (Figure 3). The connectome structure allows us to observe that indeed the remainder PNs, labeled as $(y 2, y 4-y 6, y 9, y 10)$, have stronger input inhibitory connections (dark bold red curves) than the PNs that participate in the output codes, labeled as $(y 1, y 3, y 6, y 7)$. We further observe that these strong connections are output connections of LNs, activated by RNs participating in one of the keys, labeled as $(x 1, x 3, x 6, x 7)$. This confirms that the strong inhibition of the remainder PNs is activated only when there is enough input from RNs participating in the keys. In addition, each input key activates the suppression of the other key, though less strongly than the suppression of the remainder. This is expected from the calibration matrix $W$ specification (see caption of Figure 2, and the definition of $W$ in Equation (10). The random connections between the LNs, defined by the connectivity matrix $E$, are seen in the graph as edges marked by light red color.

Once the connections are determined, the deterministic dynamics of the calibrated connectome defined in Equations (1-3) can be explored computationally in order to verify that the calibration gives the desired low-dimensional dynamics. In Figures 3B,C we depict the active pathways in the connectome, i.e., the pathways activated by the input keys. We demonstrate that for the input $\vec{J}_{1}$ (Figure 3B) four excitatory edges are activated in the connectome, where the edges from $x 1$ are stronger than from $x 3$ as expected. These edges excite LNs that activate inhibitory pathways to PNs. The strongest inhibitory pathway is invoked by $z 1$ that suppresses strongly all remainder PNs. There is also relatively strong suppression of the PNs that participate in the input key $\vec{J}_{2}$ and very weak suppression of PNs that should be activated when the input is $\vec{J}_{1}$. For the input key $\vec{J}_{2}$ (Figure 3C) the remainder is strongly suppressed again, but by a different $\mathrm{LN}$ $(z 7)$. Moreover, the suppression of neurons that should respond to $\vec{J}_{1}$ is stronger than that of $\vec{J}_{2}$, i.e., the suppression is switched as expected to support $\vec{J}_{2}$ instead of $\vec{J}_{1}$.

From the structure of the effective connectome, we can conclude that it indeed produces the expected low-dimensional dynamics. Further verification is shown in Figure 2C where the dynamics of the full network are exactly the dynamics of the prescribed projected low dimensional system, Equation (7). When $\vec{J}_{1}$ is the input, Figure 2C (left), all trajectories are attracted to a unique stable fixed point on the vertical axis, and when the input is $\vec{J}_{2}$, Figure 2C (right), the trajectory is attracted to the unique stable fixed point on the horizontal axis.

\subsection{NOISY INPUTS}

Input into the AL varies significantly as a function of time due to environmental effects, producing low signal-to-noise ratio input signals to the AL. We can use the example network as a prototype system to study the stochastic dynamics of such networks and the implications on the calibration proposed here. To simulate noisy inputs, we define the input as $\vec{J}=\alpha \vec{J}_{k}+\sigma \eta(t)$ and define the signal-to-noise ratio (SNR) as $\alpha / \sigma$. The noise $\eta(t)$ is modeled as white noise with positive normal distribution, $\eta(t) \sim|\mathcal{N}(0, \sigma)|$, and accounts in most general way for the overlap between the stimuli, overlap between RN response and other effects such as spontaneous activity, and channel noise. From recordings when the stimulus was absent or when control stimulus (mineral oil) was applied we estimated that $\sigma=0.3$.

Our objective is to verify that for different SNR ratios, the performance of the network produces the correct population encoding vector, as observed in experimental studies of the AL. To quantify the contrast enhacement, we introduce the measure, contrast over time (CRT), for a noisy input key $\vec{J}_{k}$, 


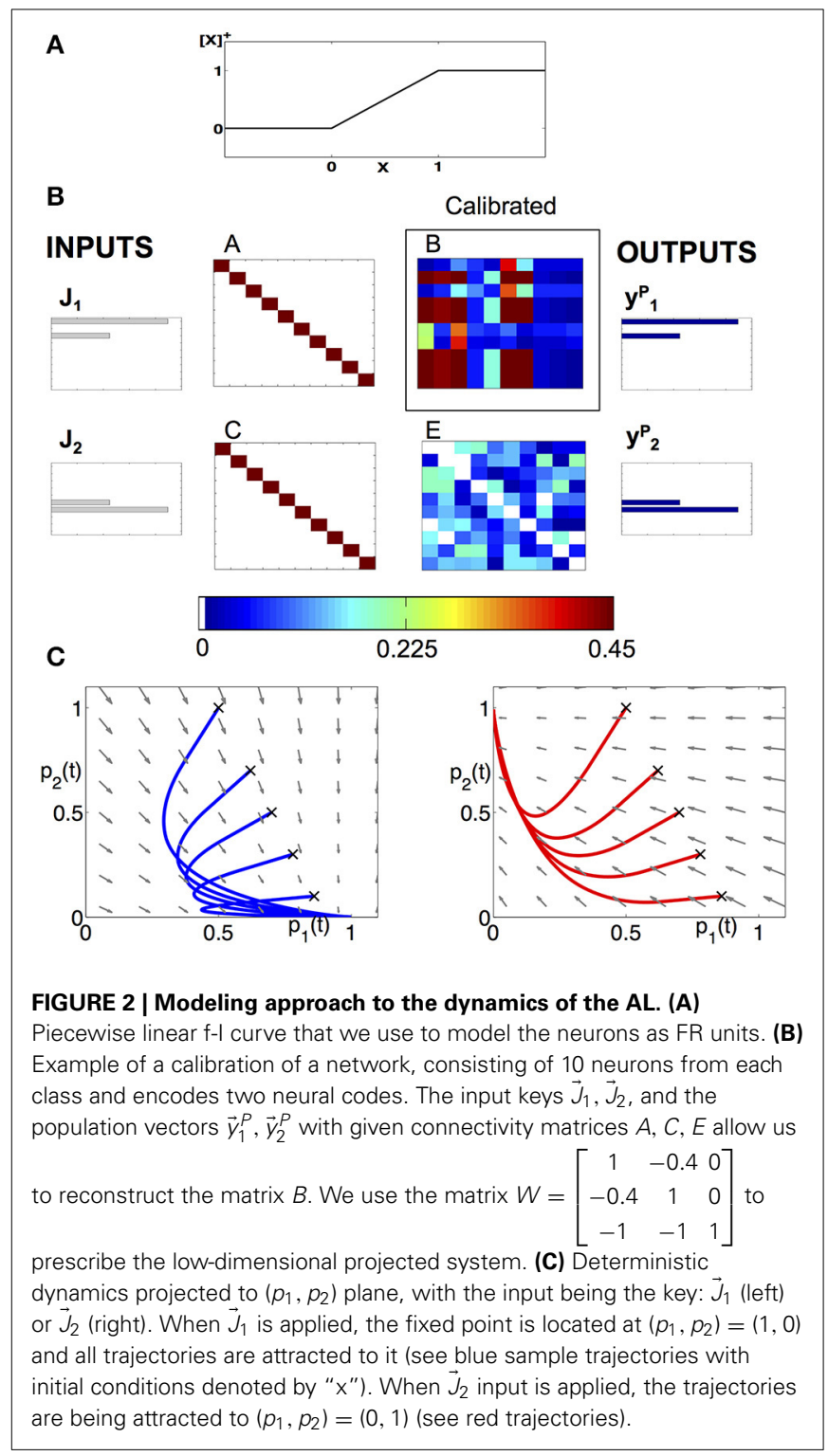

defined as $C R T_{k}=p_{k}(t)-\sum_{j=1, j \neq k}^{l} p_{j}(t)-r(t)$. This describes the difference between the $k$-th population encoding vector, $p_{k}(t)$, and the summed dynamics over all other population encoding vectors, $p_{j}(t)$, and the remainder $r(t)$.

Intuitively, the measure will be larger when there is a better separation between the correct input and all other possible inputs. We investigate the average CRT over time vs. SNR in Figure 4 (left) for three different network structures where the matrix $B$ is calibrated, uncalibrated (random with different magnitudes) and has no inhibiton (all zeros). It can be clearly observed that the calibrated network achieves the best CRT out of all other network wirings. The calibrated network exhibits a 1.5 to 4 -fold increase in CRT values in comparison to its corresponding uncalibrated networks, and a 10 -fold increase over the case where there is no inhibition. In particular, network calibration is important at low SNR rations (1.5-3.5), which is the expected noise band in the actual environment (Bhandawat et al., 2007;
Riffell et al., 2008, 2009b). Otherwise, the correct population encoding vector cannot be separated from the background noise, Figure 4A. Indeed, only the calibrated CRT curve is able to cross the 0.75 CRT threshold (approximately $75 \%$ of separation) in that SNR band. By varying the amplitude of the uncalibrated connections we illustrate that the amplitude of the elements in B do not necessarily improve the CRT. When the amplitude is low (see gray curve for 0 amplitude) the performance is poor because the activity is noisy. Incrementally increasing the amplitude improves the performance such that it is able to cross the CRT threshold when SNR exceeds 4 (red curves). However, for a calibrated network the crossing of the threshold happens for much lower values of SNR. Remarkably, even for SNR lower than 1 (where noise prevails over the signal) the calibrated CRT curve (blue) is already crossing the threshold. Additional increase in the amplitude of the inhibitory connections will inhibit both noise and the signal, and we indeed observe that the CRT curve (brown) drops lower than the lowest amplitude curves and does not cross threshold in the 0-5 SNR band.

To understand the contrast enhancement more intuitively, we show in Figure 4B the dynamics for $S N R=3$. At this $S N R$, the dynamics of RNs and LNs are very similar for all network wirings. The dynamics of RNs are noisy, making it very difficult to recover the input key from the data. The dynamics of LNs are cleaner, but still do not have a clear signature of the input key signal. In particular the ratio between the two elements of the key, neurons 1 and 3, is incorrect. The dynamics of the PNs, however, are very different for the three choices of network wirings. In the calibrated network the dynamics of PNs are more distinguishable relative to other networks. Indeed, both FRs over time and average FRs indicate that the output signal is the closest to the population encoding vector $o_{1}^{P}$ corresponding to the input key $\vec{J}_{1}$ (the CRT value is around 1). For uncalibrated or no lateral inhibition wirings, such a clear signature cannot be detected. Indeed the CRT measure for uncalibrated network is 0.55 and for no inhibition network is 0.2 .

We also compared the calibrated and uncalibrated wirings obtained from data (stimuli C and D) by adding noise of $\sigma=0.3$ $(\mathrm{SNR}=3)$ to the stimuli and computing the CRT trajectory over time for each simulation ( 5 simulations per wiring), see Figure S1. Indeed, the CRT trajectories produced by the calibrated model cross the correct threshold, i.e., they approach the correct fixed point, while trajectories produced by the random model do not cross it. In addition, when the stimulus was turned off the trajectories produced using randomly wired model became very sensitive to noise to the extent that they can cross the wrong thresholds.

\subsection{CONSTRUCTION OF THE DATA-DRIVEN MODEL}

We proceed and construct a dynamical model using the experimental data. In the first series of experiments we recorded from 130 PNs that were stimulated with two odorants: "A" (BALBombykal), "B" (MYR- $\beta$-Myrcene). These stimuli are behaviorally effective odorants: odorant $\mathrm{A}$ is a component of the moth sex pheromone, and odorant $\mathrm{B}$ is a flower scent component. These odorants excite distinct glomeruli in the AL [male sex pheromone is processed in a distinct area-the macroglomerular complex MGC (Christensen and Hildebrand, 1988; Homberg 

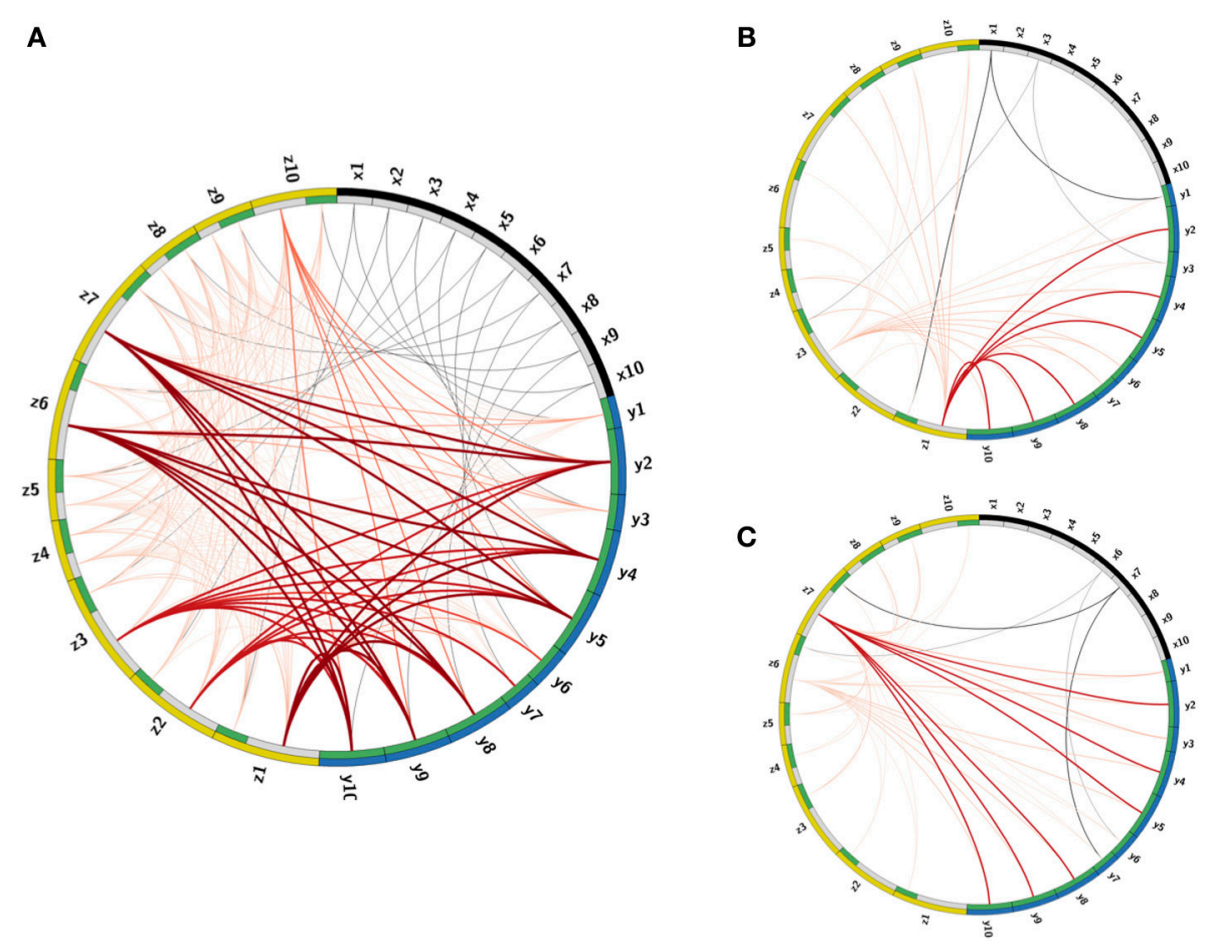

FIGURE 3 | Visualization of the Connectome. (A) The connectome (neurons and their connections) of the reconstructed network depicted in a ring shape. The nodes on the outer ring correspond to RNs, LNs, and PNs and marked by black, yellow, and blue colors, respectively. The inner ring splits each node into "in" and "out" terminals colored by gray and green colors. The edges denote connections between the nodes. Excitatory connections are displayed by gray color and inhibitory by red color. The shades and width of the edges denote the strength of the connections (darker and wider curve corresponds to a stronger connection). (B,C) Activated pathways in the connectome when the input is the key: $\vec{J}_{1}$ (B) or $\vec{J}_{2}$ (C). The shades and width of the "out" edges in the connectome scaled by the input into the node. When there is no input into the node, its "out" edge is not shown. et al., 1988; Hansson et al., 1991; Hildebrand and Shepherd, 1997)] and thus require a minimal orthogonalization of the library. Therefore, we chose them to validate the data-driven model construction part of our approach. Another reason for the choice is that they are (negatively) correlated with each otherwhen a particular stimulus is on, PNs associated with it are excited while those associated with the other stimulus are inhibited (see Figure S1). This suggests that these regions inhibit each other via lateral inhibition. We also recorded from $77 \mathrm{PNs}$ with two related stimuli: "C" (BEA-benzaldehyde), "D” (BOL-benzyl alcohol) that excite PNs in overlapping glomeruli. Both odorants are dominant in floral scents related in chemical structure as oxygenated aromatic volatiles.

The odorants are presented to the preparation at a realistic time interval $(200 \mathrm{~ms})$ repeatedly for five stimulations separated by long intervals of no input. For more information regarding the experimental setup and procedures see the subsection "Electrophysiological preparation and stimulation" in the Materials and Methods section. The data is available in the Supplementary Material. With the spike trains of each PN we have computed the time series of the instantaneous FR (iFR) averaged over the 5 trials of odor introduction. Sampling the iFR at a specific time after the beginning of the odor introduction (at $150 \mathrm{~ms}$ ) or performing a PCA reduction and taking the first dominant mode, we obtained a histogram of iFRs for the neurons for each of the odors. The neurons with substantial difference in iFR in response to the two odorants were assigned as selective neurons ( 37 neurons for A,B and 32 neurons for C,D). Those with low iFR were assigned as remainder neurons ( 60 neurons for A,B and 45 neurons for $\mathrm{C}, \mathrm{D})$. The remaining neurons that exhibited high iFR were not included in the calibration (33 neurons for A,B) since there was not enough data to calibrate the inhibitory connections to them.

Application of the orthogonalization procedure, defined in the Materials and Methods section, for the 97 neurons for A,B and 77 neurons for $\mathrm{C}, \mathrm{D}$ resulted in the two population encoding vectors: $\vec{o}_{1}^{P}$ for $\mathrm{C}$ (blue) and $\vec{o}_{2}^{P}$ for $\mathrm{D}$ (red) as shown in Figure 5 (vectors for A,B are shown in Figure S4). For A,B the required orthogonalization is minimal, while for C,D it is significant as shown in Figure S2.

This allows for the reconstruction of the connectome of the AL network in a similar fashion to the example network. Here the matrix $E$ is taken as a random normal matrix and the matrix $B$ is calibrated. The full network consists of the three populations (PNs, LNs, RNs) of 77 neurons (231 neurons in total), where in each population we depict (in the clockwise direction) the selective neurons followed by the remainder neurons (active submatrix $B$ Figure 5 left and connectome Figure 5 right). Although many connections exist, the ring shaped visualization demonstrates qualitatively the main features of the connectome: (1) the suppression of the selective neurons seems to be nonuniform and sparse while (2) the inhibition of remainder neurons 


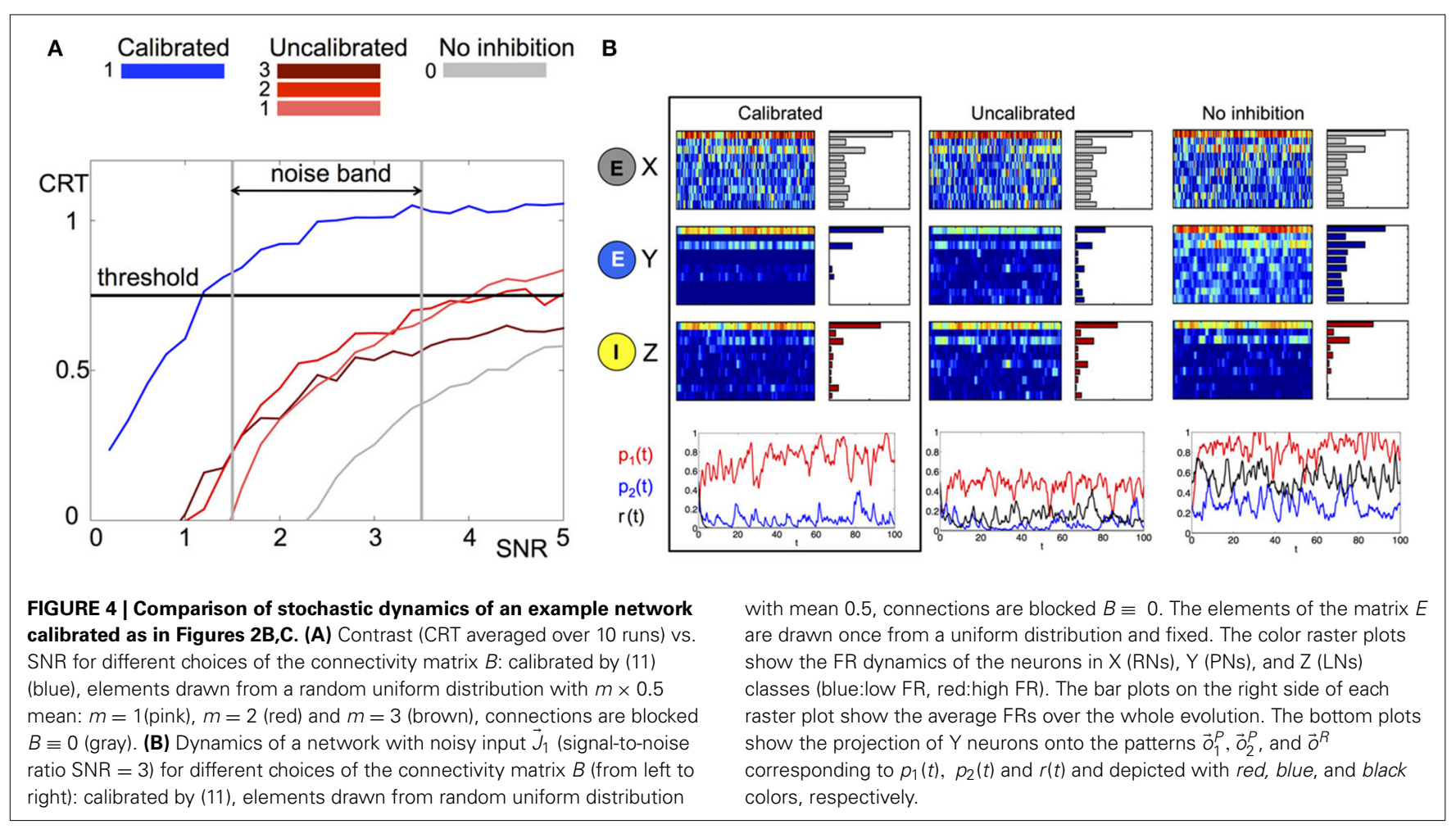

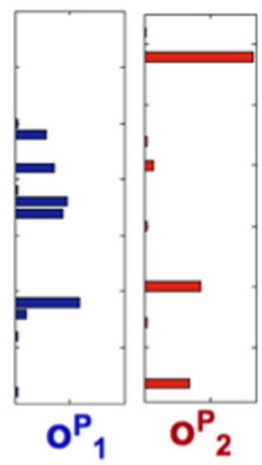

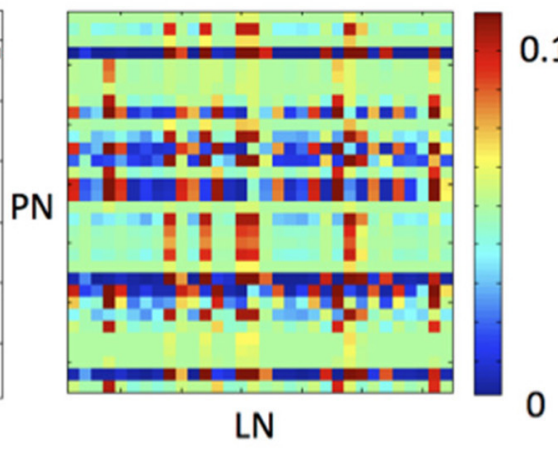

FIGURE 5 | Library elements and the reconstructed connectome produced from experimental data. Left: Part of the connectivity matrix $B$ connecting LN neurons with active PN neurons (PN-rows, LN-columns) inferred using Equation 11 for patterns that correspond to BEA and BOL (C and D) stimuli. Right: The connectome of a network of 77 neurons of each type (231 in total), obtained from solving the minimization problem in (11) with the matrices $A, C$ chosen as identity matrices and $E$ as a positive random matrix with elements drawn from a normal distribution, i.e., $e_{i j} \sim|N(0,0.02)|$. The connectome is visualized in a ring shape with similar choice of colors as in with mean 0.5 , connections are blocked $B \equiv 0$. The elements of the matrix $E$ are drawn once from a uniform distribution and fixed. The color raster plots show the FR dynamics of the neurons in $X(R N s), Y(P N s)$, and $Z$ (LNs) show the projection of $Y$ neurons onto the patterns $\vec{o}_{1}^{P}, \vec{o}_{2}^{P}$, and $\vec{o}^{R}$ colors, respectively. is uniform and dense. The non-uniformity of the wirings is consistent with the non-uniformity of the population encoding vectors.

\subsection{THE DYNAMICS OF POPULATION ENCODING VECTORS}

The orthogonality of the population encoding vectors, $\vec{o}_{1}^{P}$ and $\vec{o}_{2}^{P}$, allows us to construct a two dimensional space, called the odor space. We use it to project the iFR time series obtained

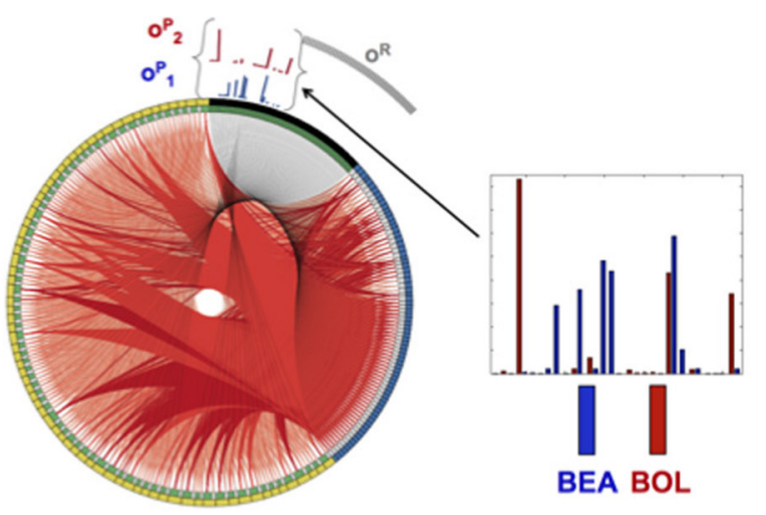

Figure 3A. The orthogonal library matrix, $O$, used in the minimization, was obtained from the electrophysiologically recorded data of two odorants BEA and $\mathrm{BOL}(\mathrm{C}$ and $\mathrm{D})$ as described in subsection Construction of the Data-Driven Model in Results section. The three library vectors $\vec{o}_{1}^{P}, \vec{o}_{2}^{P}$ and $\vec{o}^{R}$ in $O$, correspond to the odorants $\mathrm{C}$ (blue), D (red) and the remainder (gray), respectively, are depicted section as bar-plots above the connectome at the locations that correspond to RNs that evoke each of the vectors. To the right of the connectome, the bar-plots of the vectors $\vec{o}_{1}^{P}$ and $\vec{o}_{2}^{P}$ are enlarged. The threshold value, $\tau$, used to construct the library vectors, is $\tau=0.07$. 
A
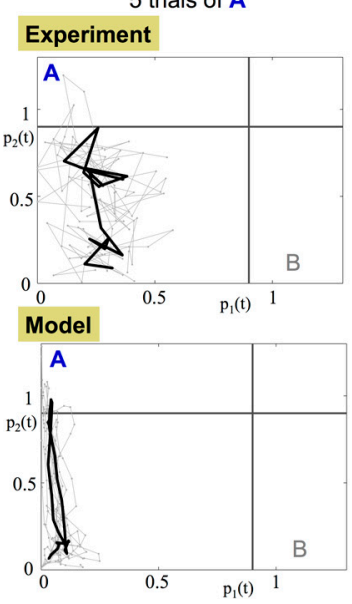

FIGURE 6 | Projection of the experimental and model dynamics onto orthogonal odor space. Top row: projected experimental dynamics of PNs. Bottom row: projected dynamics of the calibrated model. Each column shows 5 distinct trials per stimulus; (A) for A,B stimuli; (B) for C,D stimuli.
B
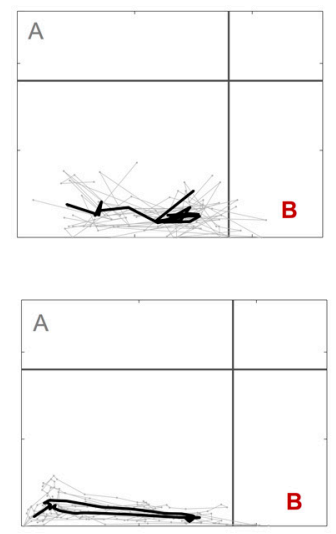

5 trials of $D$
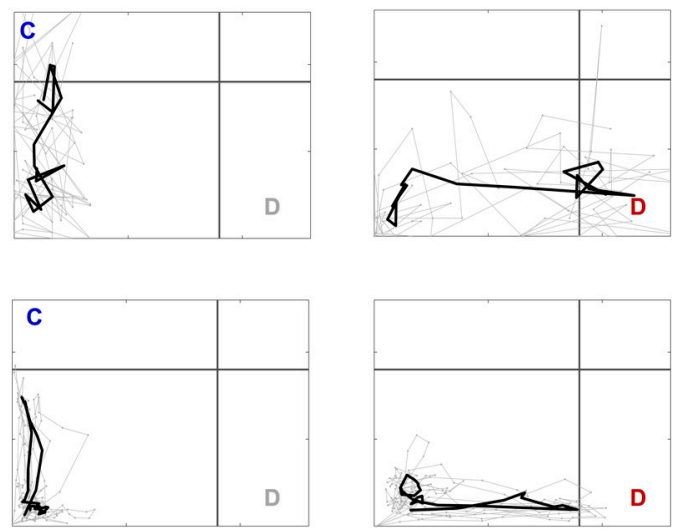

Gray trajectories are 5 distinct trials of the application of the odor. The starting and ending points of the plotted trajectories are 100 and $600 \mathrm{ms,}$ respectively, after the beginning of the trial. The black bold trajectory is the averaged trajectory over the trials.

to noise fluctuations. When the input is applied, the trajectory begins an excursion toward the stable fixed point and when the input is off, the trajectory returns to within the vicinity of the origin. The input of odor "A," "C" corresponds to trajectories whose fixed point lies on the vertical axis while odor " $\mathrm{B}$," " $\mathrm{D}$ " trajectories evolve trajectories toward a fixed point on the horizontal axis.

Data projections from both the experimental data and the calibrated model, Figure 6 and Supplementary Videos, clearly demonstrate that different odorant inputs correspond to different orthogonal fixed points in the projection space. Furthermore, trajectories appear noisy while reaching the fixed point whereupon they remain static for a while until the input is stopped and then trajectory returns to the origin.

\subsection{DECISION MAKING}

In the experiments described here, the presentation of a stimulus odor occurs for an extremely short period of time (approximately $200 \mathrm{~ms}$ ). Such inputs correspond to realistic stimulus for which the moth is flying and sampling odors in a turbulent environment. Thus, once we have characterized the dynamics of each short trial, we examine possible classifiers for odor detection and selection.

To formulate the decision making process, we analyze the dynamics of a trajectory toward the orthogonal fixed point when the stimulus is introduced as demonstrated in Figure 6. The orthogonality of the fixed points allows us to construct threshold lines for determining odor detection. The gray horizontal and vertical lines in Figure 6 represent the threshold for the detection of odor A and odor B, respectively. Application of a single odorant ensures that the dynamical trajectory crosses only a single threshold line on its way to its corresponding fixed point. Experiments show that it spends only a small amount of time near the fixed point (approximately $100 \mathrm{~ms}$ ) before returning back to the origin.

While it is difficult to measure the convergence rate of the trajectory to the fixed point, it is straightforward to detect a crossing

of the threshold line. Indeed, a common hypothesis in decision making associates crossing of a threshold in neuronal activity as equivalent to making a decision (Bogacz et al., 2006; Wong and Wang, 2006). The crossing of the threshold line in our case can be captured most effectively by computing the CRT measure, shown in Figure 7, per each trajectory of each odorant. Results from this analysis demonstrate that after the input is introduced, the CRT curve tends toward one of the decision thresholds, passes it and then returns back to the region where no clear contrast exists between odorants. Thereby, passing of the threshold creates an evidence toward one of the odorants. Integration of such evidence over several trials can produce a significant bias toward a specific odorant. When enough crossings from trial to trial occur, strong evidence is accumulated to accurately determine an odorant.

From simulations we observe that there is a clear advantage in repetitive introduction of the input in short bursts rather than a single long input. Long input, when noisy, creates a corresponding noisy trajectory that typically crosses the threshold line a single time and then wanders around the fixed point so that the decision is based only on one evidence. Other measures such as the time the trajectory spent near the fixed point are typically non-robust when noisy dynamics are considered. In contrast, repetitive inputs generate a mechanism that allows for better integration of evidence since for each trial it is enough to just cross the threshold once given by a simple measure like the CRT. Such a mechanism thus provides a rapid, robust approach to odor detection.

\section{DISCUSSION}

In this study we introduce a new method for data-driven model construction of the AL. We validate our construction with two novel extensive real multi-neuron recorded data sets, recorded for several stimuli, both related (overlapping) and orthogonal (pheromone and floral), repeated for at least five trials and applied for realistic time scales. With the constructed model 


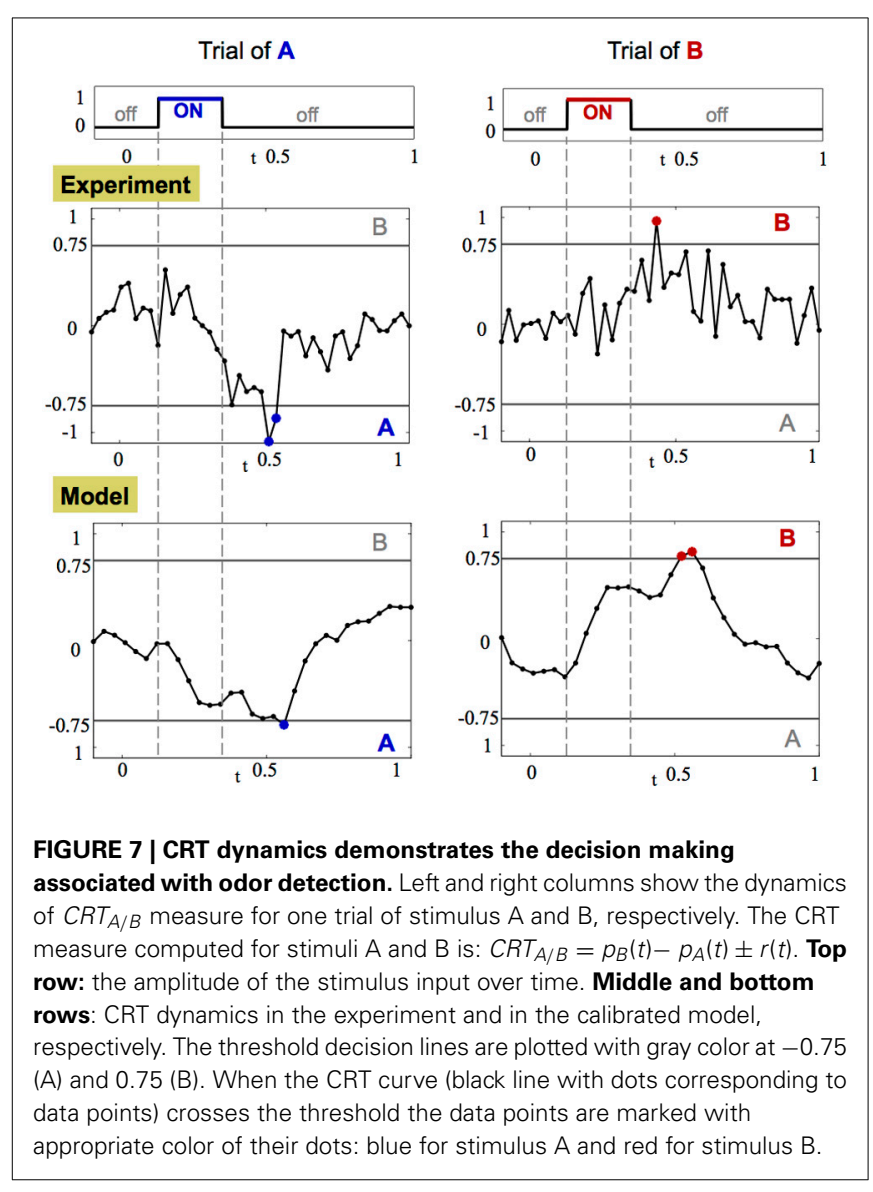

we are able to get valuable insights on the biological questions of how lateral inhibition is wired and show that calibration of wiring is required for contrast enhancement. While these questions were observed and discussed in the cited literature, (e.g., Laurent, 2002; Cleland and Linster, 2005; Mazor and Laurent, 2005; Rabinovich et al., 2006; Buckley and Nowotny, 2012; Capurro et al., 2012), here for the first time we give concrete answer on how to calibrate the network and the impact of wiring on contrast enhancement. Our results also link between independent previous experimental observations, e.g., contrast enhancement (Christie and Westbrook, 2006; Reisenman et al., 2008) with non-local wiring (Silbering and Galizia, 2007; Riffell et al., 2009a).

Contrasted with previous studies, (e.g., Afraimovich et al., 2004; Cleland and Linster, 2005; Mazor and Laurent, 2005; Buckley and Nowotny, 2012; Capurro et al., 2012), our work introduces several novel components which allow us to construct the data-driven model. The first component is that the construction of the odor space introduced here is optimal and allows for obtaining a discriminative set of orthogonal neural codes in contrast to constructions based on PCA that are limited (Galán et al., 2004; Mazor and Laurent, 2005). This basis allows us to achieve inference of the wiring from data vs. random setup used in previous works (Cleland and Linster, 2005; Buckley and Nowotny, 2012; Capurro et al., 2012). The advantage of the method introduced here is that there is no additional fitting to be done in the model and trajectories of our model, in response to stimuli, show close resemblance when compared directly with data. Prior to our approach, model generated trajectories were theoretical and could not be compared with data on neural level. We then show the important role that calibrated wiring plays in contrast enhancement, which was observed as a phenomenon in previous work but the mechanisms for it were not yet specified (Christie and Westbrook, 2006; Reisenman et al., 2008). To quantify the effect of network wiring on the dynamics of both the model and the data we define the contrast enhancement metric (CRT metric) and show that the CRT performance of the calibrated network prevails random calibrated networks. In addition, to explore the realistic transient dynamics and their relation to olfactory decision making, we apply the stimuli for short realistic times $(200 \mathrm{~ms})$ vs. much longer time scales in related work (3$5 \mathrm{~s}$ ) that showed fixed point dynamics, (e.g., Mazor and Laurent, 2005), and observe that trajectories are still able to approach the fixed points, by crossing their associated threshold lines, both in the model and data.

\subsection{HIGH-DIMENSIONAL NEURAL NETWORK IS TUNED TO EXHIBIT LOW-DIMENSIONAL DYNAMICS}

Our primary contribution is the introduction of a new methodology that combines dimensionality reduction of dynamical systems with experimental data in order to achieve a reliable computational model, that highlights the exploitation of lowdimensional encoding in the AL. To our knowledge, this is the first successful model that combines such a data-driven methodology in conjunction with dynamical equations of FR activity.

The methodology is divided into two stages. The first stage is implicit, where we define an (implicit) library of population (encoding) vectors and project the dynamic neuronal network onto these vectors (Sirovich, 1996; Shlizerman et al., 2012). The outcome is then restricted so that the system possesses stable orthogonal fixed points, where each orthogonal direction is associated with a different population encoding vector. This restriction determines a mapping from the high- to lowdimensional system. At this stage, the connectivity is kept general and expressed (implicitly) by the matrices $A, B, C, E$. As such it is generalizable to modeling the AL across different species and other neuronal networks. In particular, a similar approach could be applicable to model networks that are further downstream in olfactory processing such as the mushroom body (MB), a neuronal unit that receives input from the $\mathrm{AL}$ and associated with memory and learning of odors. Within the MB, Kenyon cells (KC) receive input from $\mathrm{PN}$ neurons and synapse to extrinsic neurons (EN). When recordings from EN become available, a similar construction can be performed to recover the connectivity of KCs to ENs (Froese et al., 2014). Furthermore, such a quasi approach (modeling both the $\mathrm{AL}$ and the $\mathrm{MB}$ ) can provide a data-driven model for testing plasticity of connectivity in the AL and $\mathrm{MB}$ and how strategies for learning-induced modulation adjust the odor space representation and the metric for decision making (Faber et al., 1999; Farooqui et al., 2003; Cassenaer and Laurent, 2012; Dacks et al., 2012; Bazhenov et al., 2013). Such modulatory effects on the encoding properties are work for future studies. The model developed here provides an efficient platform for performing such studies. 
In the second stage of the modeling, the constructed mapping is used in conjunction with experimental recordings to both determine the population encoding vectors and reconstruct the AL connectome associated with each odor in the library (see Figure 4). The construct is consistent with AL experimentally described functionality: while it is a high-dimensional neural network consisting of thousands of neurons, it appears to be tuned to exhibit low-dimensional coding dynamics.

\subsection{MODEL SETUP AND EXTENSIONS}

The model constructed here is based on experimental findings and derivation that showed that dynamics induced by stimuli converge to displaced fixed points in a low-dimensional space (Galán et al., 2004; Mazor and Laurent, 2005; Buckley and Nowotny, 2012). Lateral inhibitory connections (LN to PN), the majority of connections within the AL, are supporting these dynamics (Sachse and Galizia, 2002). Our methodology allows for inference of these inhibitory connections by deriving the minimization problem in Equation (11), where a first order approximation of fixed points is being computed by calibrating lateral inhibitory connections.

The propagation of information in the model is similar to propagation of signals in the AL. Signal propagation in the AL is initiated when the stimulus is turned ON. LN and PN dynamics are then induced by the stimulus input. In LN population, the dynamics respond to the input by releasing two GABA transmitters GABA-A (fast, order of few ms) and GABA-B (slow) (Meyer et al., 2013). The connections that are faster, GABA-A, are modeled by the matrix $E$ and correspond to the first order approximation of the fixed point. Indeed, we assume that fast GABA-A dynamics allow for quick trajectory (faster than the single $\mathrm{ON}$ duration of the stimulus) from quiescent state to the fixed point. Adding GABA-B connections would be a second order correction for the fixed point and could improve the estimate, however, it is not expected to play a significant role for the time scales of the stimulus (of $200 \mathrm{~ms}$ ) that we consider. In contrast to LN population, PN population receives two inputs: directly from the stimulus (RN to $\mathrm{LN}$ ) and from $\mathrm{LN}$ (RN to $\mathrm{LN}$ to PN), such that the timescale of PN response will be determined by these two inputs. In the model they are controlled by norms of the matrices $A, B$ and parameter $\gamma$. The pathway that involves $\mathrm{LN}$ is longer since LN is an intermediate step to reach PN. Previous works, however, show that AMPA connections in GABAergic systems can be much faster than in non-GABAergic suggesting that the two pathways could be of comparable timescales, as we assume in the calibration of the model (Geiger et al., 1997). Experimental and computational explorations of the effects of different timescales on the dynamics are left for future work.

In addition, excitatory $\mathrm{LN}$ to $\mathrm{PN}$ and $\mathrm{PN}$ to $\mathrm{PN}$ connections were found in fruit flies, honeybees, although in much smaller numbers than inhibitory LN to PN (Olsen et al., 2007; Root et al., 2007; Shang et al., 2007; Sinakevitch et al., 2013). Their presence suggests that incorporating these connections would be important in deriving a data-driven generic AL model across species. While related work is ambiguous about the impact of these connections, e.g., see Serrano et al. (2013) where the gain control condition of the AL depended only on the inhibitory connections regardless of inclusion of excitatory connections, in principle, such connections could have an impact on the stability of the fixed points and their dynamics. For example, if one of the fixed points loses stability, the dynamics then would be oscillatory or expressed by homoclinic/heteroclinic orbits (Afraimovich et al., 2004; Rabinovich et al., 2006). Even if all the fixed points remain stable, the transient dynamics could differ, e.g., the trajectories could spiral into the (stable focus) fixed point.

The data-driven construction can be extended to include excitatory connections and these different dynamics. Incorporation of excitatory connections and requiring that the dynamics are of the same features (quick dynamics to a stable fixed point) would add additional conditions to Equation (11). For example, a stability condition would restrict all the eigenvalues of the system in Equation (8) to be negative. This condition can be formulated as a Lyapunov equation, i.e., minimizing for an additional matrix, usually denoted by $Q$, that solves the Lyapunov equation and is positive (Gajic and Lelic, 1996). Furthermore, in a similar manner, by imposing pure imaginary eigenvalues in the low dimensional space, the dynamics could be set to oscillatory around some of the fixed points or unstable when the eigenvalues have real positive components.

\subsection{LATERAL INHIBITION AND CONTRAST ENHANCEMENT}

With this framework established, we are able to suggest answers to key questions in the behavior of the AL. One of primary importance is identifying the optimal network design that maximizes contrast enhancement and reproduces the observed AL functionality. The model shows that the optimal design can be constructed by tuning the lateral-inhibition so that the patterns of FR activity are made robust (Rabinovich et al., 2008). In particular, we show that asymmetric, non-local design of connections in a network of neurons can lead to such low dimensional robust functionality.

Furthermore, we demonstrate that in a noisy environment, network tuning is necessary for robust detection of an odor, even when input keys and output codes are identical. We show that lateral-inhibition, that has been tuned, shapes the noisy input into reliable and repeatable trajectories, while inhibition that was not tuned produces noisy and unreliable trajectories. This phenomenon is experimentally observed and described as contrast enhancement. Furthermore, our work suggests that absence of inhibition will result in noisier responses and scattered trajectories in the odor space. These predictions can be verified by pharmacological treatment of the AL with GABA antagonists that block inhibition.

Lateral inhibition is essential for shaping the response to complex stimuli, i.e., a mixture of odorants (Laing and Francis, 1989; Duchamp-Viret et al., 2003). Neural responses to these stimuli were shown to be of non-linear nature and phenomenologically classified into three major types: suppression (when the response to a mixture is lower than of a single odorant), hypoadditivity (response is equivalent to a dominant single odorant) and synergism (responses are magnified) (Capurro et al., 2012). The inferred wiring in our model can permit these various types of dynamics through competition. The responses are controlled via the matrix $W$ that specifies the weights of interaction between the neural codes. Higher inhibitory weights for a particular odorant indicates that the response might be of a winner-take-all type (i.e., hypoadditivity) regime. If several odorants are of comparable 
influence on inhibition then they are most likely to settle to a lower response - mutual existence type and effectively exhibit suppression type responses. For strongly overlapping stimuli we can expect to observe the effect of synergism as well-a blend of odorants can create a stronger effective stimulus into one of the axes of the odor space. Although for achieving a significant synergism excitatory LN-PN or PN-PN connections may play a role. In future studies, simulations of the model and variation of the elements of the matrix $W$ (similarly to Capurro et al., 2012 for random connectivity), in conjunction with the decision making algorithm and comparison with experimental trajectories, can reveal the regimes that odor competition produces.

\subsection{PROJECTION SPACE FOR ODOR DETECTION}

In previous studies, a three dimensional projection space (odor space) was constructed using PCA based dimension reduction. Projections of distinct odorant trajectories onto this lowdimensional space appeared to be well separated from each other (Laurent, 2002; Galán et al., 2004; Mazor and Laurent, 2005). Moreover, for each odor there was an associated fixed point that was separated from all other odor fixed points. The construction demonstrated that odorants can be classified into distinct groups and suggested that odor detection may be accomplished solely from recordings and projection onto the odor space.

The odor space is the backbone of our model as well. There are key differences, however, in the construction of our underlying odor space. Specifically, we treat the data differently by dividing the population of PNs into remainder and population encoding vectors so that we achieve a model representing the dynamics of the spatio-temporal FR patterns rather than single neurons. Such a viewpoint of the data is useful since it constructs an odor space (phase space of a dynamical system) with meaningful axes, i.e., our dimensionality reduction gives an orthogonal basis where each vector corresponds to an individual odorant (Figure S3) or a remainder (Figure 2C). As a result, the odor space provides an easy means for odor recognition and characterization.

\subsection{DECISION MAKING AS A ROBUST MECHANISM FOR ODOR PERCEPTION}

The timescales of realistic inputs indicate that odor detection occurs relatively fast and usually requires repetitive (over several trials) exposure to the same odor (Koehl et al., 2001; Mainland and Sobel, 2006). Some animals use sniffing or other mechanisms to achieve fast repetition of similar input into the olfactory system (Mafra-Neto and Carde, 1994; Vickers and Baker, 1994; Mazurek et al., 2003; Riffell et al., 2009b). Furthermore, there exists experimental evidence that shows that for a longer stimulus duration (a few seconds), initial sharp response of PNs is followed by more intermittent one (Christensen and Hildebrand, 1988; Marion-Poll and Tobin, 1992). These results suggest that the optimal strategy for scent recognition is employed by sampling the stimulus. For Manduca sexta, the optimal frequency for behavioral response to the Datura flower appears to be $1 \mathrm{~Hz}$ with the stimulus applied for $500 \mathrm{~ms}$ in each period (similar to honeybees Wright et al., 2009). Neural responses are observed to reach a maximum fixed point at about $100 \mathrm{~ms}$. These time scales indicate that there is a 5 -fold difference between neural and behavioral responses which could be due to repetitive sampling of stimulus.
Our analysis suggests that indeed based on the dynamics of the AL the exposure to multiple, short-time bursts of odor can be formulated as a decision making process. More precisely, we are able to prescribe an algorithm, possibly evoked by higher centers in the brain such as the MB or the lateral horn, that poll the dynamics of the AL in order to make a decision. Examination of the projections of iFR data produced in both theory and experiment indicates that in each short trial, the most plausible dynamical response is an excursion in odor space along a trajectory attracted toward an orthogonal fixed point. In fact, the orthogonality of the fixed points allows for an optimal separation of trajectories for different odors. Due to the short timescale of the odor burst, the trajectory does not necessarily converge to the fixed point. Rather, it only approaches its vicinity (Figure 6 and Supplementary Videos). In effect, it crosses the threshold line of an odorant while staying away from crossing thresholds of other odorants, see the horizontal and vertical lines in Figure 6 and the trajectories that cross them. Indeed, a common hypothesis in decision making is that the decision is made when neuronal activity crosses a threshold (Bogacz et al., 2006). Tracking trajectories that cross decision thresholds is accomplished by defining a linear contrast measure over time as we demonstrate in Figure 7. Repetition of the same odorant stimulus permits robustness of the algorithm. With each threshold crossing, evidence is integrated toward a specific odorant stimulus. After each trial, a probability distribution is updated until there is a high probability that supports a specific odorant stimulus. This indicates that enough evidence was integrated toward one of the odorants, and thus leads to a decision/perception for the odor, which is followed by a behavioral response corresponding to that odorant. The signature of this decision making mechanism is that integration for longer time will cease to improve accuracy. In the context of mammalian olfaction (rats) it was shown that indeed decisions do not necessarily improve with additional time (Zariwala et al., 2013). Future experiments that test behavior for various durations of stimuli may provide more evidence into olfactory decision making and its underlying mechanisms.

The proposed algorithm is scalable and can be used for perception of complex odors, i.e., a mixture of odorants (Laing and Francis, 1989; Duchamp-Viret et al., 2003). If the odorants in the compound are of similar significance and strength, then the trajectories in the odor space may cross several thresholds of distinct odorants each time that a stimulus is applied. Repeating the application of the same stimulus, eventually will lead to reconstruction of a uniform probability distribution indicative of the distribution of odorants in the mixture. Note that to obtain a reliable probability distribution the process may require many repetitions than in the detection of a single odorant.

\section{ACKNOWLEDGMENTS}

The authors would like to thank the reviewers of the manuscript for their constructive comments and suggestions. We thank E. Sanders and E. Lutz for assistance in electrophysiological experiments and J. Kunert for help with the CIRCOS software. The work was supported by the National Science Foundation under Grant No. NSF/NIGMS DMS-1361145 (Eli Shlizerman and Jeffrey A. Riffell) and by the University of Washington Royalty Research Fund (Jeffrey A. Riffell). The authors would also like to 
acknowledge the partial support by the Department of Applied Mathematics and the Department of Biology at the University of Washington.

\section{SUPPLEMENTARY MATERIAL}

The Supplementary Material for this article can be found online at: http://www.frontiersin.org/journal/10.3389/fncom. 2014.00070/abstract

\section{DATA SHARING}

Data is shared at: http://dx.doi.org/10.6084/m9.figshare.904819

\section{REFERENCES}

Afraimovich, V. S., Zhigulin, V. P., and Rabinovich, M. I. (2004). On the origin of reproducible sequential activity in neural circuits. CHAOS 14, 1123-1129. doi: $10.1063 / 1.1819625$

Bazhenov, M., Huerta, R., and Smith, B. H. (2013). A computational framework for understanding decision making through integration of basic learning rules. J. Neurosci. 33, 5686-5697. doi: 10.1523/JNEUROSCI.4145-12.2013

Bell, R. A., and Joachim, F. G. (1976). Techniques for rearing laboratory colonies of tobacco hornworms and pink bollworms lepidoptera-sphingidae-gelechiidae. Ann. Entomol. Soc. Am. 69, 365-373.

Bhandawat, V., Olsen, S. R., Gouwens, N. W., Schlief, M. L., and Wilson, R. I. (2007). Sensory processing in the Drosophila antennal lobe increases reliability and separability of ensemble odor representations. Nat. Neurosci. 10, 1474-1482. doi: 10.1038/nn1976

Bock, D. D., Lee, W.-C. A., Kerlin, A. M., Andermann, M. L., Hood, G., Wetzel, A. W., et al. (2011). Network anatomy and in vivo physiology of visual cortical neurons. Nature 471, 177-182. doi: 10.1038/nature09802

Bogacz, R., Brown, E., Moehlis, J., Holmes, P., and Cohen, J. D. (2006). The physics of optimal decision making: a formal analysis of models of performance in twoalternative forced-choice tasks. Psychol. Rev. 113, 700-765. doi: 10.1037/0033295X.113.4.700

Broome, B. M., Jayaraman, V., and Laurent, G. (2006). Encoding and decoding of overlapping odor sequences. Neuron 51, 467-482. doi: 10.1016/j.neuron.2006. 07.018

Brown, E. N., Kass, R. E., and Mitra, P. P. (2004). Multiple neural spike train data analysis: state-of-the-art and future challenges. Nat. Neurosci. 7, 456-461. doi: $10.1038 / \mathrm{nn} 1228$

Buckley, C., and Nowotny, T. (2011). Multiscale model of an inhibitory network shows optimal properties near bifurcation. Phys. Rev. Lett. 106, 238109. doi: 10.1103/PhysRevLett.106.238109

Buckley, C. L., and Nowotny, T. (2012). Transient dynamics between displaced fixed points: an alternate nonlinear dynamical framework for olfaction. Brain Res. 1434, 62-72. doi: 10.1016/j.brainres.2011.07.032

Capurro, A., Baroni, F., Olsson, S. B., Kuebler, L. S., Karout, S., Hansson, B. S., et al. (2012). Non-linear blend coding in the moth antennal lobe emerges from random glomerular networks. Front. Neuroeng. 5:6. doi: 10.3389/fneng.2012.00006

Cassenaer, S., and Laurent, G. (2012). Conditional modulation of spiketiming-dependent plasticity for olfactory learning. Nature 482, 47-52. doi: 10.1038/nature 10776

Chong, K. Y., Capurro, A., Karout, S., and Pearce, T. C. (2012). Stimulus and network dynamics collide in a ratiometric model of the antennal lobe macroglomerular complex. PLoS ONE 7:e29602. doi: 10.1371/journal.pone.0029602

Christensen, T., and Hildebrand, J. (1988). Frequency coding by central olfactory neurons in the sphinx moth Manduca sexta. Chem. Senses. 13, 123-130. doi: 10.1093/chemse/13.1.12

Christensen, T. A., Pawlowski, V. M., Lei, H., and Hildebrand, J. G. (2000). Multi-unit recordings reveal context-dependent modulation of synchrony in odor-specific neural ensembles. Nat. Neurosci. 3, 927-931. doi: 10.1038/78840

Christensen, T. A., Waldrop, B. R., and Hildebrand, J. G. (1998). Multitasking in the olfactory system: context-dependent responses to odors reveal dual GABAregulated coding mechanisms in single olfactory projection neurons. J. Neurosci. $18,5999-6008$.

Christie, J. M., and Westbrook, G. L. (2006). Lateral excitation within the olfactory bulb. J. Neurosci. 26, 2269-2277. doi: 10.1523/JNEUROSCI.4791-05.2006
Cleland, T. A., and Linster, C. (2005). Computation in the olfactory system. Chem. Senses 30, 801-813. doi: 10.1093/chemse/bji072

Cook, P. B., and McReynolds, J. S. (1998). Lateral inhibition in the inner retina is important for spatial tuning of ganglion cells. Nat. Neurosci. 1, 714-719. doi: $10.1038 / 3714$

Dacks, A. M., Riffell, J. A., Martin, J. P., Gage, S. L., and Nighorn, A. J. (2012). Olfactory modulation by dopamine in the context of aversive learning. $J$. Neurophysiol. 108, 539-550. doi: 10.1152/jn.00159.2012

Duchamp-Viret, P., Duchamp, A., and Chaput, M. A. (2003). Single olfactory sensory neurons simultaneously integrate the components of an odour mixture. Eur. J. Neurosci. 18, 2690-2696. doi: 10.1111/j.1460-9568.2003.03001.x

Egger, V., Svoboda, K., and Mainen, Z. F. (2003). Mechanisms of lateral inhibition in the olfactory bulb: efficiency and modulation of spike-evoked calcium influx into granule cells. J. Neurosci. 23, 7551-7558.

Faber, T., Joerges, J., and Menzel, R. (1999). Associative learning modifies neural representations of odors in the insect brain. Nat. Neurosci. 2, 74-78. doi: $10.1038 / 4576$

Farooqui, T., Robinson, K., Vaessin, H., and Smith, B. H. (2003). Modulation of early olfactory processing by an octopaminergic reinforcement pathway in the honeybee. J. Neurosci. 23, 5370-5380. Available online at: http://www.jneurosci.org/content/23/12/5370.short

Froese, A., Szyszka, P., and Menzel, R. (2014). Effect of gabaergic inhibition on odorant concentration coding in mushroom body intrinsic neurons of the honeybee. J. Comp. Physiol. A 200, 183-195. doi: 10.1007/s00359-013-0877-8

Gajic, Z., and Lelic, M. (1996). Modern Control Systems Engineering. Upper Saddle River, NJ. Prentice-Hall, Inc.

Galán, R. F., Sachse, S., Galizia, C. G., and Herz, A. V. (2004). Odor-driven attractor dynamics in the antennal lobe allow for simple and rapid olfactory pattern classification. Neural Comput. 16, 999-1012. doi: 10.1162/089976604773135078

Galizia, C. G., and Menzel, R. (2000). Odour perception in honeybees: coding information in glomerular patterns. Curr. Opin. Neurobiol. 10, 504-510. doi: 10.1016/S0959-4388(00)00109-4

Geiger, J. R., Lübke, J., Roth, A., Frotscher, M., and Jonas, P. (1997). Submillisecond ampa receptor-mediated signaling at a principal neuron-interneuron synapse. Neuron 18, 1009-1023. doi: 10.1016/S0896-6273(00)80339-6

Grant, M., and Boyd, S. (2011). CVX: Matlab Software for Disciplined Convex Programming. Version 1.21. Available online at: http://cvxr.com/cvx

Hansson, B. S., and Anton, S. (2000). Function and morphology of the antennal lobe: new developments. Annu. Rev. Entomol. 45, 203-231. doi: 10.1146/ annurev.ento.45.1.203

Hansson, B. S., Christensen, T. A., and Hildebrand, J. G. (1991). Functionally distinct subdivisions of the macroglomerular complex in the antennal lobe of the male sphinx moth manduca sexta. J. Comp. Neurol. 312, 264-278. doi: $10.1002 /$ cne. 903120209

Harris, K. D., Bartho, P., Chadderton, P., Curto, C., de la Rocha, J., Hollender, L., et al. (2011). How do neurons work together? Lessons from auditory cortex. Hear. Res. 271, 37-53. doi: 10.1002/cne.903120209

Hildebrand, J. G., and Shepherd, G. M. (1997). Mechanisms of olfactory discrimination: converging evidence for common principles across phyla. Annu. Rev Neurosci. 20, 595-631. doi: 10.1146/annurev.neuro.20.1.595

Homberg, U., Montague, R., and Hildebrand, J. (1988). Anatomy of antennocerebral pathways in the brain of the sphinx moth manduca sexta. Cell Tissue Res. 254, 255-281. doi: 10.1007/BF00225800

Hopfield, J., and Tank, D. (1986). Computing with neural circuits: a model. Science 233, 625-633. doi: 10.1126/science.3755256

Jbabdi, S., and Behrens, T. E. J. (2012). Specialization: the connections have it. Nat. Neurosci. 15, 171-172. doi: 10.1126/science.3755256

Koehl, M. A. R., Koseff, J. R., Crimaldi, J. P., McCay, M. G., Cooper, T., Wiley, M. B., et al. (2001). Lobster sniffing: antennule design and hydrodynamic filtering of information in an odor plume. Science 294, 1948-1951. doi: 10.1126/science. 1063724

Krzywinski, M., Schein, J., Birol, I., Connors, J., Gascoyne, R., Horsman, D., et al. (2009). Circos: an information aesthetic for comparative genomics. Genome Res. 19, 1639-1645. doi: 10.1101/gr.092759.109

Laing, D. G., and Francis, G. (1989). The capacity of humans to identify odors in mixtures. Physiol. Behav. 46, 809-814. doi: 10.1016/0031-9384(89)90041-3

Laughlin, B. Y. S. B., and Osorio, D. (1989). Mechanisms for neural signal enhancement in the blowfly compound eye. J. Exp. Biol. 146, 113-146.

Laurent, G. (1999). A systems perspective on early olfactory coding. Science 286, 723-728. doi: $10.1126 /$ science. 286.5440 .723 
Laurent, G. (2002). Olfactory network dynamics and the coding of multidimensional signals. Nat. Rev. Neurosci. 3, 884-895. doi: 10.1038/nrn964

Lei, H., Reisenman, C. E., Wilson, C. H., Gabbur, P., and Hildebrand, J. G. (2011). Spiking patterns and their functional implications in the antennal lobe of the tobacco hornworm Manduca sexta. PLoS ONE 6:e23382. doi: 10.1371/journal.pone. 0023382

Linster, C., Sachse, S., and Galizia, C. G. (2005). Computational modeling suggests that response properties rather than spatial position determine connectivity between olfactory glomeruli. J. Neurophysiol. 93, 3410-3417. doi: 10.1152/jn.01285.2004

Mafra-Neto, A., and Carde, R. T. (1994). Fine-scale structure of pheromone plumes modulates upwind orientation of flying moths. Nature 369, 142-144. doi: $10.1038 / 369142 \mathrm{a} 0$

Mainland, J., and Sobel, N. (2006). The sniff is part of the olfactory percept. Chem. Senses 31, 181-196. doi: 10.1093/chemse/bjj012

Marion-Poll, F., and Tobin, T. (1992). Temporal coding of pheromone pulses and trains in Manduca sexta. J. Comp. Physiol. A. 171, 505-512. doi: 10.1007/BF00194583

Martin, J. P., Beyerlein, A., Dacks, A. M., Reisenman, C. E., Riffell, J. A., Lei, H., et al. (2011). The neurobiology of insect olfaction: sensory processing in a comparative context. Prog. Neurobiol. 95, 427-447. doi: 10.1016/j.pneurobio.2011.09.007

Mazor, O., and Laurent, G. (2005). Transient dynamics versus fixed points in odor representations by locust antennal lobe projection neurons. Neuron 48 , 661-673. doi: 10.1016/j.neuron.2005.09.032

Mazurek, M., Roitman, J., Ditterich, J., and Shadlen, M. (2003). A role for neural integrators in perceptual decision making. Cereb. Cortex 13, 1257-1269. doi: 10.1093/cercor/bhg097

Meyer, A., Galizia, C. G., and Nawrot, M. P. (2013). Local interneurons and projection neurons in the antennal lobe from a spiking point of view. J. Neurophysiol. 110, 2465-2474. doi: 10.1152/jn.00260.2013

Murils, J., and Jones, C. D. (1981). Fine-scale structure of odor plumes in relation to insect orientation to distant pheromone and other attractant sources. Physiol. Entomol. 6, 71-86. doi: 10.1111/j.1365-3032.1981.tb00262.x

Nagel, K. I., and Wilson, R. I. (2011). Biophysical mechanisms underlying olfactory receptor neuron dynamics. Nat. Neurosci. 14, 208-216. doi: 10.1038/nn.2725

Olsen, S. R., Bhandawat, V., and Wilson, R. I. (2007). Excitatory interactions between olfactory processing channels in the drosophila antennal lobe. Neuron 54, 89-103. doi: 10.1016/j.neuron.2007.03.010

Olsen, S. R., and Wilson, R. I. (2008). Lateral presynaptic inhibition mediates gain control in an olfactory circuit. Nature 452, 956-960. doi: 10.1038/nature06864

Rabinovich, M., Huerta, R., and Laurent, G. (2008). Neuroscience. Transient dynamics for neural processing. Science 321, 48-50. doi: 10.1126/science.1155564

Rabinovich, M., Volkovskii, A., Lecanda, P., Huerta, R., Abarbanel, H., and Laurent, G. (2001). Dynamical encoding by networks of competing neuron groups: winnerless competition. Phys. Rev. Lett. 87:068102. doi: 10.1103/PhysRevLett.87. 068102

Rabinovich, M. I., Huerta, R., and Varona, P. (2006). Heteroclinic synchronization: ultrasubharmonic locking. Phys. Rev. Lett. 96:014101. doi: 10.1103/PhysRevLett.96.014101

Reisenman, C. E., Heinbockel, T., and Hildebrand, J. G. (2008). Inhibitory interactions among olfactory glomeruli do not necessarily reflect spatial proximity. $J$. Neurophysiol. 100, 554-564. doi: 10.1152/jn.90231.2008

Riffell, J. A., Abrell, L., and Hildebrand, J. G. (2008). Physical processes and realtime chemical measurement of the insect olfactory environment. J. Chem. Ecol. 34, 837-853. doi: 10.1007/s10886-008-9490-7

Riffell, J. A., Lei, H., Abrell, L., and Hildebrand, J. G. (2013). Neural basis of a pollinators buffet: olfactory specialization and learning in Manduca sexta. Science 339, 200-204. doi: 10.1126/science. 1225483

Riffell, J. A., Lei, H., Christensen, T. A., and Hildebrand, J. G. (2009a). Characterization and coding of behaviorally significant odor mixtures. Curr. Biol. 19, 335-340. doi: 10.1016/j.cub.2009.01.041

Riffell, J. A., Lei, H., and Hildebrand, J. G. (2009b). Neural correlates of behavior in the moth Manduca sexta in response to complex odors. Proc. Natl. Acad. Sci. U.S.A. 106, 19219-19226. doi: 10.1073/pnas.0910592106

Root, C. M., Semmelhack, J. L., Wong, A. M., Flores, J., and Wang, J. W. (2007). Propagation of olfactory information in drosophila. Proc. Natl. Acad. Sci. U.S.A. 104, 11826-11831. doi: 10.1073/pnas.0704523104
Sachse, S., and Galizia, C. G. (2002). Role of inhibition for temporal and spatial odor representation in olfactory output neurons: a calcium imaging study. $J$. Neurophysiol. 87, 1106-1117.

Serrano, E., Nowotny, T., Levi, R., Smith, B. H., and Huerta, R. (2013). Gain control network conditions in early sensory coding. PLoS Comput. Biol. 9:e1003133. doi: 10.1371/journal.pcbi.1003133

Seung, H. S. (2011). Neuroscience: towards functional connectomics. Nature 471, 170-172. doi: 10.1038/471170a

Shang, Y., Claridge-Chang, A., Sjulson, L., Pypaert, M., and Miesenböck, G. (2007). Excitatory local circuits and their implications for olfactory processing in the fly antennal lobe. Cell 128, 601-612. doi: 10.1016/j.cell.2006.12.034

Shlizerman, E., Schroder, K., and Kutz, J. N. (2012). Neural activity measures and their dynamics. SIAM J. Appl. Math. 72, 1260-1291. doi: 10.1137/110843630

Silbering, A. F., and Galizia, C. G. (2007). Processing of odor mixtures in the drosophila antennal lobe reveals both global inhibition and glomerulus-specific interactions. J. Neurosci. 27, 11966-11977. doi: 10.1523/JNEUROSCI.309907.2007

Sinakevitch, I. T., Smith, A. N., Locatelli, F., Huerta, R., Bazhenov, M., and Smith, B. H. (2013). Apis mellifera octopamine receptor 1 (amoa1) expression in antennal lobe networks of the honey bee (apis mellifera) and fruit fly (drosophila melanogaster). Front. Syst. Neurosci. 7:70. doi: 10.3389/fnsys.2013.00070

Sirovich, L. (1987). Turbulence and the dynamics of coherent structures. I Coherent structures. Q. Appl. Math. 45, 561-571, 573-590.

Sirovich, L. (1996). Modeling the functional organization of the visual cortex. Physica D Nonlinear Phenom. 96, 355-366. doi: 10.1016/0167-2789(96)00033-4

Stopfer, M., and Laurent, G. (1999). Short-term memory in olfactory network dynamics. Nature 402, 664-668. doi: 10.1038/45244

Tumlinson, J., Brennan, M., Doolittle, R., Mitchell, E., Brabham, A., Mazomenos, B., et al. (1989). Identification of a pheromone blend attractive to Manduca sexta (L.) males in a wind tunnel. Arch. Insect. Biochem. Physiol. 271, 255-271. doi: 10.1002/arch.940100402

Tumlinson, J., Mitchell, E., Doolittle, R., and Jackson, D. (1994). Field-tests of synthetic manduca-sexta sex-pheromone. J. Chem. Ecol. 20, 579-591. doi: 10.1007/BF02059599

Vickers, N. J., and Baker, T. C. (1994). Reiterative responses to single strands of odor promote sustained upwind flight and odor source location by moths. Proc. Natl. Acad. Sci. U.S.A. 91, 5756-5760. doi: 10.1073/pnas.91.13.5756

Wilson, R. I. (2008). Neural and behavioral mechanisms of olfactory perception. Curr. Opin. Neurobiol. 18, 408-412. doi: 10.1016/j.conb.2008.08.015

Wong, K. F., and Wang, X. J. (2006). A recurrent network mechanism of time integration in perceptual decisions. J. Neurosci. 26, 1314-1328. doi: 10.1523/JNEUROSCI.3733-05.2006

Wright, G. A., Carlton, M., and Smith, B. H. (2009). A honeybee's ability to learn, recognize, and discriminate odors depends upon odor sampling time and concentration. Behav. Neurosci. 123, 36. doi: 10.1037/a0014040

Yokoi, M., Mori, K., and Nakanishi, S. (1995). Refinement of odor molecule tuning by dendrodendritic synaptic inhibition in the olfactory bulb. Proc. Natl. Acad. Sci. U.S.A. 92, 3371-3375. doi: 10.1073/pnas.92.8.3371

Zariwala, H. A., Kepecs, A., Uchida, N., Hirokawa, J., and Mainen, Z. F. (2013). The limits of deliberation in a perceptual decision task. Neuron 78, 339-351. doi: 10.1016/j.neuron.2013.02.010

Conflict of Interest Statement: The authors declare that the research was conducted in the absence of any commercial or financial relationships that could be construed as a potential conflict of interest.

Received: 21 January 2014; accepted: 20 June 2014; published online: 13 August 2014. Citation: Shlizerman E, Riffell JA and Kutz JN (2014) Data-driven inference of network connectivity for modeling the dynamics of neural codes in the insect antennal lobe. Front. Comput. Neurosci. 8:70. doi: 10.3389/fncom.2014.00070 This article was submitted to the journal Frontiers in Computational Neuroscience. Copyright (๑) 2014 Shlizerman, Riffell and Kutz. This is an open-access article distributed under the terms of the Creative Commons Attribution License (CC BY). The use, distribution or reproduction in other forums is permitted, provided the original author(s) or licensor are credited and that the original publication in this journal is cited, in accordance with accepted academic practice. No use, distribution or reproduction is permitted which does not comply with these terms. 